\title{
The Gaia reference frame for bright sources examined using VLBI observations of radio stars
}

\author{
Lennart Lindegren \\ Lund Observatory, Department of Astronomy and Theoretical Physics, Lund University, Box 43, 22100 Lund, Sweden \\ e-mail: lennart@astro.lu.se
}

Received 24 June 2019 / Accepted 22 October 2019

\begin{abstract}
Context. Positions and proper motions of Gaia sources are expressed in a reference frame that ideally should be non-rotating relative to distant extragalactic objects, coincident with the International Celestial Reference System (ICRS), and consistent across all magnitudes. For sources fainter than 16th magnitude, this is achieved through Gaia's direct observations of quasars. At brighter magnitudes, it is difficult to validate the quality of the reference frame because comparison data are scarce.

Aims. The aim of this paper is to examine the use of very long baseline interferometry (VLBI) observations of radio stars to determine the spin and orientation of the bright reference frame of current and future Gaia data releases.

Methods. Simultaneous estimation of the six spin and orientation parameters makes optimal use of VLBI data and makes it possible to include even single-epoch VLBI observations in the solution. The method is applied to Gaia Data Release 2 (DR2) using published VLBI data for 41 radio stars.

Results. The VLBI data for the best-fitting 26 sources indicate that the bright reference frame of Gaia DR2 rotates relative to the faint quasars at a rate of about 0.1 mas yr$^{-1}$, which is significant at the $2 \sigma$ level. This supports a similar conclusion based on a comparison with stellar positions in the HIPPARCos frame. The accuracy is currently limited because only a few radio sources are included in the solution, by uncertainties in the Gaia DR2 proper motions, and by issues related to the astrophysical nature of the radio stars.

Conclusions. While the origin of the indicated rotation is understood and can be avoided in future data releases, it remains important to validate the bright reference frame of Gaia by independent observations. This can be achieved using VLBI astrometry, which may require re-observing the old sample of radio stars as well as measuring new objects. The unique historical value of positional measurements is stressed and VLBI observers are urged to ensure that relevant positional information is preserved for the future.
\end{abstract}

Key words. astrometry - proper motions - reference systems - instrumentation: interferometers - methods: data analysis

\section{Introduction}

The Gaia Celestial Reference Frame (Gaia-CRF; Gaia Collaboration 2018a) is formally defined by the positions, as measured by Gaia, of a large number of sources that are identified as quasars. Through their cosmological distances, these objects define a kinematically non-rotating reference frame, that is, their proper motions are assumed to be zero on average. A subset of them, identified as the optical counterparts of radio sources with accurate positions in the International Celestial Reference Frame (ICRF; Ma et al. 1998) from very long baseline interferometry (VLBI) observations, are used to align the axes of the non-rotating quasar frame with the ICRF. The second release of Gaia data (DR2; Gaia Collaboration 2018b) lists 556869 quasars whose positions at epoch J2015.5 define the optical reference frame known as Gaia-CRF2. This includes 2820 sources matched to a prototype version of ICRF3 (Jacobs et al. 2018).

The vast majority of sources in Gaia DR2 are Galactic stars with sizeable proper motions. The implicit assumption is that the positions and proper motions of the stars, and indeed the barycentric coordinates of all Gaia sources, are expressed in the same reference frame as the quasars. This is fundamental for the dynamical interpretation of the observations, which assumes the absence of the inertial (Coriolis and centrifugal) forces that would appear in a rotating frame.
Although the measurement and reduction principles of the Gaia mission have been designed to provide a globally consistent reference frame for all kinds of objects, subtle differences are inevitable as a consequence of the varying conditions under which the objects are observed. For example, the quasars defining Gaia-CRF are all faint (fewer than $1 \%$ have $G<17 \mathrm{mag}$ ), on average bluer than stars of comparable magnitude, and they have a very different distribution on the sky than the stars. Differences in magnitude, colour, and numerous other factors are likely to produce small shifts of the image centroids, which, if left uncalibrated, may propagate into systematic errors of the positions and proper motions.

Intricate instrument models have been set up and calibrated as part of the global astrometric reductions of Gaia data in order to eliminate such systematics. In Gaia DR2 there is nevertheless an indication that the reference frame defined by the bright stars (up to $G \simeq 11-13$ ) rotates with respect to the quasars at a rate of a few tenths of a milliarcsecond (mas) per year. This is illustrated in Fig. 4 of Lindegren et al. (2018) and further quantified by Brandt (2018). In Brandt's paper the rotation shows up as a systematic offset of the proper motions of bright stars in Gaia DR2 with respect to the "HIPPARCOS-Gaia proper motions" calculated from the position differences between Gaia DR2 (at epoch 2015.5) and the HIPPARCos catalogue (at epoch 1991.25), scaled by the epoch difference of $\sim 24 \mathrm{yr}$. Through the long time-baseline, the HIPPARCos-Gaia proper motions constitute a 


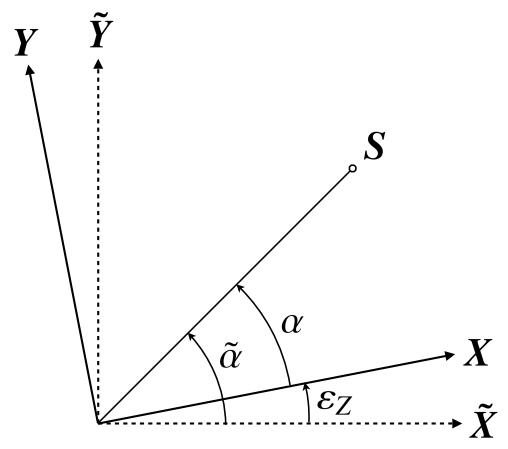

Fig. 1. Convention for the definition of the orientation of the arbitrary frame $\tilde{C}=[\tilde{\boldsymbol{X}} \tilde{\boldsymbol{Y}} \tilde{\boldsymbol{Z}}]$ with respect to the $\operatorname{ICRF}(\mathrm{C}=[\boldsymbol{X} \boldsymbol{Y} \boldsymbol{Z}])$. The drawing illustrates the configuration when the orientation difference is a pure rotation about the $Z$ axes by the positive angle $\varepsilon_{Z}$, i.e. $\varepsilon=\left[0,0, \varepsilon_{Z}\right]^{\prime}$. The right ascension of the source at $S$ is $\alpha$ in frame $\mathrm{C}$ and $\tilde{\alpha}=\alpha+\varepsilon_{Z}$ in frame $\tilde{C}$.

precise set of reference values, which are moreover inertial because the positional systems of HIPPARCOS and Gaia were both aligned with the International Celestial Reference System (ICRS) at their respective epoch. The observed offset therefore points to a systematic error in the Gaia DR2 proper motions of the bright stars, equivalent to an inertial rotation of its reference frame.

The cause of this rotation is discussed in Appendix B. Briefly, it is related to the different modes in which Gaia's CCDs are operated, depending on the magnitude of the source, and corresponding differences in the calibration models. In particular, around $G=13$, there is a transition in the on-board CCD sampling scheme from two- to one-dimensional pixel windows, causing abrupt changes in the quality of both the astrometric data and the $G$-band photometry (which uses the same CCDs). Examples of this are shown in Figs. 9 and B.2 of Lindegren et al. (2018), and Fig. 9 of Evans et al. (2018).

It is therefore justified to study the reference frames separately that are defined by the bright and the faint Gaia sources, and to draw the division line at $G \simeq 13$. Gaia-CRF2, being defined by the quasars, clearly belongs to the faint part and is by construction to very high accuracy non-rotating with respect to the ICRS; its properties are discussed elsewhere (Gaia Collaboration 2018a). The subject of this paper is the bright reference frame of Gaia, defined by the system of proper motions for sources with $G \lesssim 13$. Available data, including the HIPPARCOS-Gaia proper motions mentioned above, are not sufficient to decide if the transition from the faint to the bright reference frame occurs abruptly at this magnitude, or more gradually over a few magnitudes; for the purpose of this paper, I will in general assume that the transition is abrupt so that all sources brighter than $G=13.0$ are in the same reference frame.

Future Gaia data releases may provide proper motions that are an order of magnitude more precise than they were in DR2, and similar or even greater improvements could be obtained in the systematics. The quasars, of which many more will be found, will continue to be the main tool for examining the quality of the Gaia reference frame at faint magnitudes. Assessing its consistency at brighter magnitudes will be much harder. The HIPPARCos-Gaia proper motions will then be of little use because their random and systematic errors are already dominated by the position errors in the HIPPARCos celestial reference frame (HCRF), which remain unchanged. The quality of the bright reference frame can only be verified by means of the positions and proper motions of bright Gaia sources, measured to sufficient accuracy in the ICRF frame by some independent method. Most obviously, this can be done by means of differential VLBI, where the positions of radio stars are measured relative to quasars by phase-referencing techniques (Lestrade et al. 1990; Beasley \& Conway 1995; Rioja \& Dodson 2011; Fomalont 2012). These relative measurements are already reaching microarcsecond ( $\mu$ as) precision (Reid \& Honma 2014).

The purpose of this paper is to explore the use of differential VLBI observations of radio stars for verification of Gaia's bright reference frame. In Sect. 2 the required formalism is developed, whereby the positions and proper motions measured by VLBI are connected to the Gaia data. The method is tested on Gaia DR2 data in Sect. 3, using a selection of published VLBI observations, and the results and possible future improvements are discussed in Sect. 4.

\section{Theory}

\subsection{ICRS and celestial reference frames}

The ICRS is an idealised system of astronomical coordinates $\alpha$, $\delta$, whose axes are defined by convention and remain fixed with respect to distant matter in the Universe (Arias et al. 1995). The origin is at the Solar System barycentre. Any astrometric catalogue where the positions and proper motions nominally refer to the ICRS can be regarded as a practical realisation of the idealised system, and is then called a celestial reference frame (CRF). The ICRF, HCRF, and Gaia-CRF2 are examples of such reference frames, among which the ICRF has the privileged status of actually defining the conventional axes of the ICRS. In the present context it is necessary to consider that Gaia DR2 may represent (at least) two distinct reference frames, one defined by the faint quasars, and a second defined by the positions and proper motions of the bright stars in Gaia DR2.

Conceptually, a CRF can be visualised as a set of orthogonal unit vectors $\boldsymbol{X}, \boldsymbol{Y}, \boldsymbol{Z}$, with origin at the Solar System barycentre, and with $\boldsymbol{X}$ pointing towards $\alpha=\delta=0, \boldsymbol{Z}$ towards $\delta=+90^{\circ}$, and $\boldsymbol{Y}=\boldsymbol{Z} \times \boldsymbol{X}$. Let $\mathrm{C}=[\boldsymbol{X} \boldsymbol{Y} \boldsymbol{Z}]$ be the vector triad representing the ICRF. By definition, $C$ coincides with the axes of the ICRS and is fixed with respect to objects at cosmological distances such as the quasars. This means that the proper motions of quasars, when expressed in $\mathrm{C}$, have no global component that can be interpreted as a solid-body rotation (spin) of C. Any other reference frame $\tilde{\mathrm{C}}=[\tilde{\boldsymbol{X}} \tilde{\boldsymbol{Y}} \tilde{\boldsymbol{Z}}]$ may have some small time-dependent offset from $\mathrm{C}$ described by the vector $\boldsymbol{\varepsilon}(t)$,

$\mathrm{C}=\tilde{\mathrm{C}}+\boldsymbol{\varepsilon}(t) \times \tilde{\mathrm{C}}+O\left(\varepsilon^{2}\right)$.

Thus $\boldsymbol{\varepsilon}(t)$ is the rotation of $\tilde{\mathrm{C}}$ needed to align its axes with $\mathrm{C}$. The sign of $\boldsymbol{\varepsilon}(t)$ is chosen for consistency with earlier publications (Lindegren \& Kovalevsky 1995; Lindegren et al. 2012, 2016), where the frame offset was defined in the sense of a correction to the frame under investigation. The components of $\boldsymbol{\varepsilon}(t)$ in $\mathrm{C}$ or $\tilde{C}$ are denoted $\varepsilon_{X}(t), \varepsilon_{Y}(t), \varepsilon_{Z}(t)$. The relation between the two frames is illustrated in Fig. 1.

Equation (1) is valid in the small-angle approximation, that is, ignoring terms of order $\varepsilon^{2}$, where $\varepsilon=|\varepsilon|$ is the total angular offset between the two frames. This is a valid approximation in all practical cases, where $\varepsilon \lesssim 1$ mas, or $\varepsilon^{2}<5 \times 10^{-9}$ mas (5 pas). Rigorous expressions are given in Sect. 6.1.2 of Lindegren et al. (2012).

The relation between the two frames can alternatively be expressed by means of the rotation matrix

$\mathrm{C}^{\prime} \tilde{\boldsymbol{C}}=\left[\begin{array}{ccc}\boldsymbol{X}^{\prime} \tilde{\boldsymbol{X}} & \boldsymbol{X}^{\prime} \tilde{\boldsymbol{Y}} & \boldsymbol{X}^{\prime} \tilde{\boldsymbol{Z}} \\ \boldsymbol{Y}^{\prime} \tilde{\boldsymbol{X}} & \boldsymbol{Y}^{\prime} \tilde{\boldsymbol{Y}} & \boldsymbol{Y}^{\prime} \tilde{\boldsymbol{Z}} \\ \boldsymbol{Z}^{\prime} \tilde{\boldsymbol{X}} & \boldsymbol{Z}^{\prime} \tilde{\boldsymbol{Y}} & \boldsymbol{Z}^{\prime} \tilde{\boldsymbol{Z}}\end{array}\right]=\left[\begin{array}{ccc}1 & \varepsilon_{Z} & -\varepsilon_{Y} \\ -\varepsilon_{Z} & 1 & \varepsilon_{X} \\ \varepsilon_{Y} & -\varepsilon_{X} & 1\end{array}\right]+O\left(\varepsilon^{2}\right)$, 
where the prime $\left({ }^{\prime}\right)$ denotes matrix transposition and the scalar product of vectors (Murray 1983). Let $\boldsymbol{u}$ be the unit vector at a certain time from the Solar System barycentre towards a celestial object. The rectangular coordinates of the vector in the two frames are given by

$\mathrm{C}^{\prime} \boldsymbol{u}=\left[\begin{array}{c}\cos \alpha \cos \delta \\ \sin \alpha \cos \delta \\ \sin \delta\end{array}\right], \quad \tilde{\mathbf{C}}^{\prime} \boldsymbol{u}=\left[\begin{array}{c}\cos \tilde{\alpha} \cos \tilde{\delta} \\ \sin \tilde{\alpha} \cos \tilde{\delta} \\ \sin \tilde{\delta}\end{array}\right]$,

where $(\alpha, \delta)$ and $(\tilde{\alpha}, \tilde{\delta})$ are the astronomical coordinates of the object in the two frames. The column matrices in Eq. (3) are related by the matrix equation

$\mathrm{C}^{\prime} \boldsymbol{u}=\left(\mathrm{C}^{\prime} \tilde{\mathrm{C}}\right) \tilde{\mathrm{C}}^{\prime} \boldsymbol{u}$,

where $C^{\prime} \tilde{C}$ is the rotation matrix in Eq. (2). To verify Eq. (4), one can note that $\tilde{C} \tilde{C}^{\prime}$ is the unit tensor (Murray 1983), and hence $\tilde{\mathrm{C}} \tilde{C}^{\prime} \boldsymbol{u}=\boldsymbol{u}$.

In frame $\tilde{C}$ the proper motions are modelled as essentially constant angular velocities, which does not permit $\tilde{C}$ to have a non-uniform rotation with respect to distant matter. The variation of the offset vector with time $t$ can therefore be written

$\boldsymbol{\varepsilon}(t)=\boldsymbol{\varepsilon}(T)+(t-T) \omega$,

where $T$ is an arbitrary reference epoch, and $\varepsilon(T)$ and $\omega$ are constant vectors with components $\varepsilon_{X}(T), \omega_{X}$, etc.

Equation (5) describes a uniform solid rotation of one frame relative to the other. The word "rotation" is ambiguous in this context because it may refer to either the instantaneous configuration $\boldsymbol{\varepsilon}(t)$ or the angular velocity $\omega$. In the following we use "spin" for the angular velocity and "orientation" for the instantaneous configuration, specifically $\varepsilon(T)$; for brevity, the combined or general effect may be called "rotation".

\subsection{Differences in position and proper motion}

From Eqs. (2)-(4), the following first-order expressions are obtained for the coordinate differences:

$(\alpha-\tilde{\alpha}) \cos \delta=+\varepsilon_{X} \cos \alpha \sin \delta+\varepsilon_{Y} \sin \alpha \sin \delta-\varepsilon_{Z} \cos \delta$,

$\delta-\tilde{\delta}=-\varepsilon_{X} \sin \alpha+\varepsilon_{Y} \cos \alpha$.

The time derivative of the above gives the corresponding expressions for the proper motion differences,

$\mu_{\alpha *}-\tilde{\mu}_{\alpha *}=+\omega_{X} \cos \alpha \sin \delta+\omega_{Y} \sin \alpha \sin \delta-\omega_{Z} \cos \delta$,

$\mu_{\delta}-\tilde{\mu}_{\delta}=-\omega_{X} \sin \alpha+\omega_{Y} \cos \alpha$,

where $\mu_{\alpha *}=(\mathrm{d} \alpha / \mathrm{d} t) \cos \delta, \mu_{\delta}=\mathrm{d} \delta / \mathrm{d} t$ are the components of the proper motion in frame $\mathrm{C}$ and $\tilde{\mu}_{\alpha^{*}}=(\mathrm{d} \tilde{\alpha} / \mathrm{d} t) \cos \tilde{\delta}, \tilde{\mu}_{\delta}=\mathrm{d} \tilde{\delta} / \mathrm{d} t$ the components in $\tilde{\mathrm{C}}$. In the small-angle approximation, one can use either set of coordinates, $(\alpha, \delta)$ or $(\tilde{\alpha}, \tilde{\delta})$, for the trigonometric factors in (6)-(9); the choice made here is arbitrary.

The use of equations such as Eqs. (6)-(9) for estimating the difference in orientation and spin between two astrometric catalogues has been well established in the literature for many years (among many others, e.g., Fricke 1977; Froeschle \& Kovalevsky 1982; Arias et al. 1988; Brosche et al. 1991; Lestrade et al. 1995; Zhu 2000; Metz \& Geffert 2004; Fedorov et al. 2011; Bobylev $2015)$. To estimate the difference in spin $(\omega)$, the typical procedure has been to set up Eqs. (8) and (9), using the proper motion differences for a number of sources with accurate proper motions in both catalogues (or for which the true proper motions can be assumed to be negligible), and solve the resulting overdetermined system of equations using the least-squares method. It is thus possible to estimate $\omega$ without knowing $\varepsilon$ because the differences in $\alpha$ and $\delta$ between the two catalogues are of second order in Eqs. (8) and (9).

The orientation difference $(\boldsymbol{\varepsilon})$ can be estimated by applying a similar procedure to the position differences, resulting in a set of equations like Eqs. (6) and (7) for the three unknowns $\varepsilon_{X}(T)$, $\varepsilon_{Y}(T)$, and $\varepsilon_{Z}(T)$. If the sources have proper motion, a complication is that the position differences must be computed for a fixed common epoch $(T)$, which may require one or both sets of positions to be propagated from their mean epoch of observation. This propagation must in addition take into account any difference in spin between the two catalogues. Except when both catalogues contain only sources with zero proper motion, it is therefore usually not possible to estimate $\varepsilon(T)$ independently of $\omega$.

The general procedure should consequently consider the joint estimation of $\varepsilon(T)$ and $\omega$. This may in fact lead to a much better determination of $\omega$ than if only proper motion differences are used. The details of the procedure are worked out below, but the basic idea is simple enough: if a set of independent positional differences are obtained for a range of epochs, the resulting Eqs. (6) and (7) will depend on both $\boldsymbol{\varepsilon}(T)$ and $\omega$, allowing all six parameters to be determined. In the current context, this means that positional VLBI observations of Gaia sources, suitably spread out in time, will contribute to the determination of the spin. This is true even when there is only a single epoch of VLBI data per source, so that their proper motions (and parallaxes) cannot be determined purely from the VLBI observations.

The realisation that positional observations contribute to the determination of the spin is of course not new. It was implicit in several of the methods used to link the HIPPARCos catalogue to the ICRS (Kovalevsky et al. 1997), and explicitly discussed by Walter \& Sovers (2000, Chap. 7.4), who concluded that it might become desirable to revise the HIPPARCos link if, in the future, many more radio stars obtained accurate interferometric positions.

\subsection{Joint estimation of the rotation parameters}

The joint estimation of $\boldsymbol{\varepsilon}(T)$ and $\boldsymbol{\omega}$ from the Gaia and VLBI data for a certain set $S$ of common sources is now considered. It is assumed that the Gaia observations refer to frame $\tilde{\mathrm{C}}$ and the VLBI observations to C (=ICRS), with Eqs. (1) and (5) connecting the frames. For conciseness, the six unknown rotation parameters are written as the column matrix $\boldsymbol{x}=$ $\left[\varepsilon_{X}(T), \varepsilon_{Y}(T), \varepsilon_{Z}(T), \omega_{X}, \omega_{Y}, \omega_{Z}\right]^{\prime}$. The result is an estimate of $\boldsymbol{x}$, denoted $\hat{\boldsymbol{x}}$, together with its $6 \times 6$ covariance matrix. As long as the full covariance of $\hat{\boldsymbol{x}}$ is retained, the choice of $T$ is in principle arbitrary, and for convenience, we adopt the same reference epoch as for the Gaia data, for example $T=\mathrm{J} 2015.5$ for Gaia DR2.

The six rotation parameters in $\boldsymbol{x}$ are not the only unknowns of the problem. Both the VLBI and the Gaia observations provide information on the astrometric parameters of the sources in the common set $S$, and a basic assumption is that each source $i$ in $S$ can only have one set of "true" astrometric parameters, here denoted by the column matrix $\boldsymbol{y}_{i}$. Considering $m=|S|$ sources, the end result of the estimation process consists of $\hat{\boldsymbol{x}}$ and the $m$ estimates $\hat{\boldsymbol{y}}_{i}$ for $i=1 \ldots m$. Effectively, each $\hat{\boldsymbol{y}}_{i}$ is the weighted mean of the astrometric parameters as determined by VLBI and by Gaia, after correcting the latter values for the estimated frame rotation $\hat{\boldsymbol{x}}$.

As discussed in Sect. 2.4, the comparison of Gaia and VLBI measurements is potentially affected by a number of difficulties including radio-optical offsets and non-linear motions. In order 
to proceed with the theoretical development, these difficulties are ignored here. It is assumed that the optical and radio data refer to the same physical point source, and that this source moves through space at uniform velocity relative to the Solar System barycentre. Astrometrically, then, the radio star is completely described by the usual five parameters $\alpha, \delta, \varpi$ (parallax), $\mu_{\alpha *}$, and $\mu_{\delta}$, referred to the adopted epoch $T$, and the radial velocity $v_{\mathrm{r}}$, assumed to be known from spectroscopy. These parameters describe the "true" motion of the source in frame C, and differ in general both from the Gaia parameters and from those derived from the VLBI measurements. The subsequent treatment is vastly simplified if expressions are linearised around a fixed set of reference values, which is an acceptable approximation as the differences are typically much smaller than an arcsecond for a suitable choice of reference values (cf. the discussion in Sect. 2.1).

It is convenient to use the astrometric parameters as given by Gaia as reference values for the linearisation. The parameter array for source $i$,

$\boldsymbol{y}_{i}=\left[\begin{array}{l}\Delta \alpha *_{i} \\ \Delta \delta_{i} \\ \Delta \varpi_{i} \\ \Delta \mu_{\alpha * i} \\ \Delta \mu_{\delta i}\end{array}\right]$

thus consists of corrections to be added to the Gaia parameters. With $m$ sources, the total number of parameters to estimate is $6+$ $5 \mathrm{~m}$. The estimation is done using a weighted least-squares algorithm, using as "observations" the Gaia data, hereafter denoted $\boldsymbol{g}_{i}$, and the VLBI measurements, denoted $\boldsymbol{f}_{i}$. We now proceed to detail how these observations depend on the unknowns.

The general model of the Gaia data is

$\boldsymbol{g}_{i}=\boldsymbol{G}_{i}\left(\boldsymbol{x}, \boldsymbol{y}_{i}\right)+\boldsymbol{\gamma}_{i}$,

where $\boldsymbol{G}_{i}$ is a function mapping the model parameters to the expected Gaia data, according to the model, and $\gamma_{i}$ is the noise. In the standard five-parameter model, $\boldsymbol{g}_{i}$ and $\boldsymbol{\gamma}_{i}$ are $5 \times 1$ column matrices. It is assumed that the Gaia data are unbiased, except for the frame rotation, and that the uncertainties are correctly represented in the Gaia catalogue, so that

$E\left[\gamma_{i}\right]=\mathbf{0}$ and $E\left[\gamma_{i} \gamma_{i}^{\prime}\right]=C_{i}$,

where $\boldsymbol{C}_{i}$ is the $5 \times 5$ covariance matrix of the Gaia parameters for source $i$. Expressing both $\boldsymbol{y}_{i}$ and $\boldsymbol{g}_{i}$ differentially with respect to the Gaia values, we have $\boldsymbol{g}_{i}=\mathbf{0}$ and the linearised version of Eq. (11) becomes

$\mathbf{0}=\boldsymbol{y}_{i}-\boldsymbol{K}_{i} \boldsymbol{x}+\gamma_{i}$,

where

$\boldsymbol{K}_{i}=\left[\begin{array}{cccccc}c \alpha_{i} s \delta_{i} & s \alpha_{i} s \delta_{i} & -c \delta_{i} & 0 & 0 & 0 \\ -s \alpha_{i} & c \alpha_{i} & 0 & 0 & 0 & 0 \\ 0 & 0 & 0 & 0 & 0 & 0 \\ 0 & 0 & 0 & c \alpha_{i} s \delta_{i} & s \alpha_{i} s \delta_{i} & -c \delta_{i} \\ 0 & 0 & 0 & -s \alpha_{i} & c \alpha_{i} & 0\end{array}\right]$

is the matrix containing the trigonometric factors from Eqs. (6)-(9). The third row of the matrix is zero because the parallax is unaffected by the frame rotation.

Although the linearised form of Eq. (13), with $\operatorname{dim}\left(\boldsymbol{y}_{i}\right)=5$, is used for the rest of this paper, the more general expression, Eq. (11), should be retained for future reference, when the Gaia observations may provide additional parameters (Sect. 2.4).
The description of the VLBI data for source $i$ is similarly written in the general form

$\boldsymbol{f}_{i}=\boldsymbol{F}_{i}\left(\boldsymbol{y}_{i}\right)+\boldsymbol{v}_{i}$

where $\boldsymbol{F}_{i}$ is a function mapping the source parameters to the expected VLBI data. The rotation parameters $\boldsymbol{x}$ do not enter here because the VLBI measurements are assumed to be in the ICRF frame. The VLBI measurement errors are represented by the column matrix $v_{i}$, with

$E\left[v_{i}\right]=\mathbf{0}$ and $E\left[v_{i} v_{i}^{\prime}\right]=V_{i}$

and known covariance matrix $\boldsymbol{V}_{i}$. The dimension of $\boldsymbol{f}_{i}$ is $n_{i} \times 1$, where $n_{i}$ depends on the number of VLBI measurements and their state of reduction. If the measurements have been reduced to a set of five astrometric parameters, similar to the Gaia data but referring to some epoch $t_{i}$ chosen specifically for these observations, we have $n_{i}=5$. The VLBI data could also consist of a single measurement of the topocentric position at epoch $t_{i}$, however, in which case $n_{i}=2$; or of a sequence of topocentric positions at different epochs. In either case, $\boldsymbol{F}_{i}\left(\boldsymbol{y}_{i}\right)$ involves a propagation of the source parameters from the reference epoch $T$ to the specific epoch(s) of the VLBI data $t_{i}$. For the standard fiveparameter astrometric model this propagation should be done as described in Appendix A.

Recalling that $\boldsymbol{y}_{i}=\mathbf{0}$ represents the source parameters according to Gaia, we see that $\Delta \boldsymbol{f}_{i}=\boldsymbol{f}_{i}-\boldsymbol{F}_{i}(\mathbf{0})$ contains the differences between the actually observed VLBI data $\boldsymbol{f}_{i}$ and the values $\boldsymbol{F}_{i}(\mathbf{0})$ computed by propagating the Gaia parameters to the VLBI epoch. To first order in $\boldsymbol{y}_{i}$, the linearised version of Eq. (15) is therefore

$\Delta f_{i}=M_{i} y_{i}+v_{i}$

where $\boldsymbol{M}_{i}=\partial \boldsymbol{F}_{i} / \partial \boldsymbol{y}_{i}^{\prime}$ is the Jacobian matrix evaluated at $\boldsymbol{y}_{i}=\mathbf{0}$. If $\boldsymbol{f}_{i}$ consists of the standard $n_{i}=5$ astrometric parameters, taken in the same order as in Eq. (10) but referring to epoch $t_{i}$, then the Jacobian is approximately given by

$\boldsymbol{M}_{i} \simeq\left[\begin{array}{ccccc}1 & 0 & 0 & t_{i}-T & 0 \\ 0 & 1 & 0 & 0 & t_{i}-T \\ 0 & 0 & 1 & 0 & 0 \\ 0 & 0 & 0 & 1 & 0 \\ 0 & 0 & 0 & 0 & 1\end{array}\right]$

This expression is accurate to first order in the total proper motion over the time interval $t_{i}-T$, which in some cases could amount to many arcseconds. Because it is then not obvious that Eq. (18) is a sufficiently good approximation, it is advisable to evaluate $\boldsymbol{M}_{i}$ by numerical differentiation of the propagation formulae.

The generalised least-squares estimate is obtained by minimising the loss function

$$
\begin{aligned}
Q\left(\boldsymbol{x},\left\{\boldsymbol{y}_{i}\right\}_{S}\right)= & \sum_{i \in S}\left[\left(\boldsymbol{y}_{i}-\boldsymbol{K}_{i} \boldsymbol{x}\right)^{\prime} \boldsymbol{C}_{i}^{-1}\left(\boldsymbol{y}_{i}-\boldsymbol{K}_{i} \boldsymbol{x}\right)\right. \\
& \left.+\left(\boldsymbol{\Delta} \boldsymbol{f}_{i}-\boldsymbol{M}_{i} \boldsymbol{y}_{i}\right)^{\prime} \boldsymbol{V}_{i}^{-1}\left(\boldsymbol{\Delta} \boldsymbol{f}_{i}-\boldsymbol{M}_{i} \boldsymbol{y}_{i}\right)\right] .
\end{aligned}
$$

On the assumption of Gaussian errors, the likelihood function is proportional to $\exp (-Q / 2)$, and minimising $Q$ is then equivalent to a maximum-likelihood estimation.

Setting the partial derivative of $Q$ with respect to each model parameter equal to zero gives the symmetric system of $6+5 m$ 
linear equations, known as the normal equations,

$$
\begin{aligned}
& \left(\sum_{i \in S} \boldsymbol{K}_{i}^{\prime} \boldsymbol{C}_{i}^{-1} \boldsymbol{K}_{i}\right) \boldsymbol{x}-\sum_{i \in S} \boldsymbol{K}_{i}^{\prime} \boldsymbol{C}_{i}^{-1} \boldsymbol{y}_{i}=\mathbf{0}, \\
& -\boldsymbol{C}_{i}^{-1} \boldsymbol{K}_{i} \boldsymbol{x}+\left(\boldsymbol{C}_{i}^{-1}+\boldsymbol{M}_{i}^{\prime} \boldsymbol{V}_{i}^{-1} \boldsymbol{M}_{i}\right) \boldsymbol{y}_{i}=\boldsymbol{M}_{i}^{\prime} \boldsymbol{V}_{i}^{-1} \boldsymbol{\Delta} \boldsymbol{f}_{i}, \quad i \in S .
\end{aligned}
$$

These can be solved by standard numerical methods, and the inverse of the normal matrix provides an estimate of the covariance of the model parameters.

Computationally, the solution of Eqs. (20) and (21) is unproblematic as it involves only a moderate number of unknowns. In terms of numerical accuracy, it is advantageous to compute the least-squares solution using orthogonal transformations (e.g. Björck 1996) after transforming the observation equations, Eqs. (13) and (17), to an equivalent set of uncorrelated unit-weight equations. Details of this procedure are not given here.

While the least-squares problem is thus solved, there is some additional insight to be gained by further manipulation of Eqs. (19)-(21). Using Eq. (21), it is possible to write each $\boldsymbol{y}_{i}$ in terms of $\boldsymbol{x}$; inserting these into Eq. (20) yields a reduced system of normal equations involving only the common parameters ${ }^{1}$,

$$
\left(\sum_{i \in S} N_{i}\right) \boldsymbol{x}=\sum_{i \in S} \boldsymbol{b}_{i}
$$

where

$$
\begin{aligned}
& \boldsymbol{N}_{i}=\boldsymbol{K}_{i}^{\prime} \boldsymbol{M}_{i}^{\prime} \boldsymbol{D}_{i}^{-1} \boldsymbol{M}_{i} \boldsymbol{K}_{i}, \\
& \boldsymbol{b}_{i}=\boldsymbol{K}_{i}^{\prime} \boldsymbol{M}_{i}^{\prime} \boldsymbol{D}_{i}^{-1} \boldsymbol{\Delta} \boldsymbol{f}_{i},
\end{aligned}
$$

and

$\boldsymbol{D}_{i}=\boldsymbol{V}_{i}+\boldsymbol{M}_{i} \boldsymbol{C}_{i} \boldsymbol{M}_{i}^{\prime}$.

Solving Eq. (22) yields $\hat{\boldsymbol{x}}$, and then $\hat{\boldsymbol{y}}_{i}$ from the $m$ Eqs. (21). Clearly, this solution is mathematically the same as obtained directly from Eqs. (20) and (21). It is more remarkable that the covariance of $\hat{\boldsymbol{x}}$ (the upper left $6 \times 6$ submatrix of the inverse of the full normal matrix) is obtained from the reduced system as $\left(\sum_{i \in S} N_{i}\right)^{-1}$.

By a similar process of eliminating the unknowns $\boldsymbol{y}_{i}$, the loss function Eq. (19) can be written in terms of $\boldsymbol{x}$ as

$Q(\boldsymbol{x})=\sum_{i \in S} Q_{i}(\boldsymbol{x})$

with

$$
Q_{i}(\boldsymbol{x})=\left(\Delta \boldsymbol{f}_{i}-\boldsymbol{M}_{i} \boldsymbol{K}_{i} \boldsymbol{x}\right)^{\prime} \boldsymbol{D}_{i}^{-1}\left(\Delta \boldsymbol{f}_{i}-\boldsymbol{M}_{i} \boldsymbol{K}_{i} \boldsymbol{x}\right) .
$$

The interpretation of the above equations is straightforward. $\Delta f_{i}-M_{i} K_{i} \boldsymbol{x}$ is the residual of the VLBI data with respect to the values predicted from the Gaia data, after correcting for the rotation parameters and propagating to the VLBI epoch. $\boldsymbol{D}_{i}$ in (25) is the covariance of $\Delta f_{i}$, including the contributions from the uncertainties of both the VLBI and propagated Gaia data. Equation (27) shows that $\hat{\boldsymbol{x}}$ minimises the sum of the squares of the VLBI residuals after normalisation by the combined uncertainties. For a given solution $\hat{\boldsymbol{x}}$, we may take the quantity

$Q_{i} / n_{i}=Q_{i}(\hat{\boldsymbol{x}}) / \operatorname{dim}\left(\boldsymbol{f}_{i}\right)$

1 This procedure, known as Helmert blocking after the German geodesist F. R. Helmert, who described the method in 1880 (Wolf 1978), is frequently applied to large-scale least-squares problems in various branches of science, including astrometry (e.g. de Vegt \& Ebner 1974). as a measure of the discrepancy for source $i$, where $n_{i}=\operatorname{dim}\left(f_{i}\right)$ is the number of VLBI data points included for the source. The normalisation by $n_{i}$ is essential in order to avoid penalising sources with many VLBI data points. $Q_{i} / n_{i}$ can be interpreted as the reduced chi-square of the source, and should ideally be around unity if the astrometric model fits the source and the uncertainties are correctly estimated. $Q_{i} / n_{i}$ is hereafter referred to as the "discrepancy measure" of the source relative to a given solution.

Equations (22)-(27) have some practical advantages over the use of orthogonal transformations to solve the least-squares problem. For the identification of outliers (Sect. 2.4), computing the solution and other statistics for a very large number of different subsets of $S$ may be required. This can be done most efficiently by pre-computing $\boldsymbol{D}_{i}, \boldsymbol{N}_{i}, \boldsymbol{b}_{i}$, and other quantities that do not depend on the solution.

The matrices $\boldsymbol{N}_{i}$ are, furthermore, useful for quantifying the amount of (Fisher) information on $\boldsymbol{x}$ contributed by each source. A source without VLBI data would formally have infinite $\boldsymbol{V}_{i}$ and hence $\boldsymbol{N}_{i}=\mathbf{0}$. A source with only proper motion data from VLBI will not contribute to the estimation of $\boldsymbol{\varepsilon}$ and will consequently have zeros in the first three rows and columns of $\boldsymbol{N}_{i}$. More generally, the amount of information contributed by source $i$ to the estimation of $\varepsilon(T)$ and $\omega$ is quantified by

$E_{i}=\underset{\varepsilon}{\operatorname{trace}}\left(N_{i}\right)$ and $\Omega_{i}=\underset{\omega}{\operatorname{trace}}\left(\boldsymbol{N}_{i}\right)$,

respectively, where the trace is limited to the first three diagonal elements of $N_{i}$ for $E_{i}$, and to the last three for $\Omega_{i}$.

\subsection{Modelling issues and robustness}

In the preceding treatment it was assumed that the sources move through space with uniform velocity relative to the Solar System barycentre, allowing both the Gaia and VLBI measurements to be accurately modelled by five astrometric parameters per source, plus a spectroscopically determined radial velocity. This model is manifestly incorrect for a number of radio stars known to be members of binaries or more complex systems, for which the VLBI observations have determined non-linear motions (e.g. the T Tau system; Loinard et al. 2007) or even complete orbits (e.g. the Algol and UX Arietis systems; Peterson et al. 2011). A second assumption is that the centre of radio emission coincides with that of the optical emission, which is also not true for many objects with extended atmospheres, discs, jets, and other structures in the radio and/or optical images. The astrometric biases produced by these various effects range over many decades, from the undetectable to tens of milliarcseconds. As a result, the simple modelling described above will provide excellent fits for some sources and large residuals for others, with a continuum of intermediate cases.

The general method of estimation in Sect. 2.3 does permit the application of more sophisticated source models. Depending on the physical nature of a radio star, it may be possible to improve the modelling, and ultimately the accuracy of $\hat{\boldsymbol{x}}$, by introducing a small number of additional unknowns, thus extending the array $\boldsymbol{y}_{i}$ in Eq. (10). For example, in an interacting binary it may be possible to model the offsets of the optical and radio emissions from the barycentre of the system in terms of a few geometrical elements if the main characteristics of the binary, including its period, are known from spectroscopy. Another example is the non-linear motion of a radio star perturbed by a distant companion. In this case, the comparison of radio and optical observations, taken at different epochs, is meaningful only if the 
non-linearity is taken into account through the addition of a few acceleration terms or possibly a complete set of orbital elements. In either case, the modelling requires an augmented parameter array $\boldsymbol{y}_{i}$ and more elaborate expressions for the functions $\boldsymbol{G}_{i}$ and $\boldsymbol{F}_{i}$ in Eqs. (11) and (15) than discussed in Sect. 2.3. However, the details of this are beyond the scope of this paper, where the standard model of Appendix A is used throughout.

To cope with unmodelled effects, whether they are radiooptical offsets, non-linear motions, or deviations from more elaborate models, it is imperative that the estimation procedure is robust, that is, that the result is not overly sensitive to the relatively few cases with large perturbations. The least-squares estimation of Sect. 2.3 is inherently non-robust and needs to be modified or complemented with other techniques to provide the required robustness. The strategy adopted in this paper is to identify the most problematic sources and exclude them from the solution. Consistent with the treatment in Sect. 2.3, each source with all its data is regarded as an independent entity, to be either included or rejected. Although it could happen that the model fits the data very well in one coordinate (say, $\alpha$ ), but not in the other $(\delta)$, a reasonable standpoint is that such a source is better left out entirely. Discrepant sources can be identified by means of statistics such as Eq. (28), and by comparing solutions for different subsets of $S$. Details of the procedure are explained in Sect. 3.3 as it is applied to actual data.

\section{Application to Gaia DR2 data}

In this section we use the algorithms in Sect. 2.3 to estimate the rotation parameters of the bright Gaia DR2 reference frame, based on VLBI astrometry of radio stars collected from the literature. The primary goal is to verify, if possible, the spin detected from comparison with HIPPARCos data (Sect. 1), but an important secondary goal is to illustrate the usefulness of positional VLBI data for estimating the spin, compared with a solution using only proper motions.

\subsection{VLBI data}

Recent technological advances have dramatically expanded the scope for stellar radio astrophysics (Matthews 2019). The amount of accurate VLBI astrometry that could potentially be used for validating the stellar reference frame of Gaia increases rapidly, not least thanks to a number of surveys aiming to study Galactic structure (e.g. BeSSeL, Brunthaler et al. 2011; VERA, Honma 2013; GOBELINS, Ortiz-León et al. 2018). A recent comparison of Gaia DR2 and VLBI stellar parallaxes (Xu et al. 2019) lists more than 100 targets.

The 41 objects considered in this study are listed in Table 1, with their corresponding Gaia DR2 identifiers in Table 2. All the sources are brighter than $G=13.0$ and have full (five-parameter) astrometry in Gaia DR2. The list includes most radio stars from the early programmes, in particular, Lestrade et al. (1999), in view of their potentially high weight in the estimation of the spin components. These were complemented by a selection of more recent data mainly from the GOBELINS survey, which have observation epochs close to the Gaia DR2 epoch and therefore could contribute usefully to the estimation of the frame orientation at J2015.5. Most of the objects are young stellar objects, interacting binaries, or giants with extended atmospheres. Their celestial distribution is shown in Fig. 2.

The VLBI data have been collected from some 20 different publications as listed in Table 1. In some cases, the authors did not publish the barycentric position at mean epoch obtained

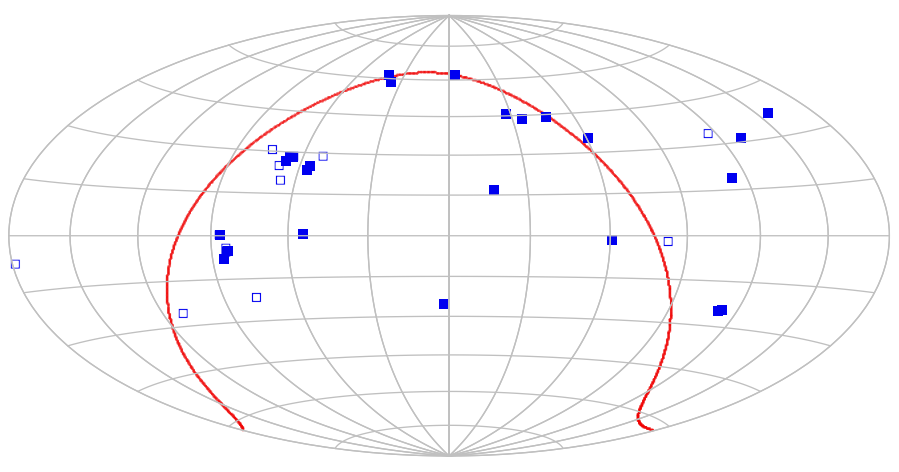

Fig. 2. Sky distribution of the radio sources in Tables 1 and 2. Filled and open symbols mark the objects that were accepted and rejected in the baseline solution. The map is a Hammer-Aitoff projection in ICRS coordinates with $\alpha=\delta=0$ in the centre, $\alpha$ increasing from right to left, and the Galactic equator is plotted in red.

in their analysis of the observations. In most of these cases, the authors did provide results from their individual VLBI sessions, however, including the dates and geocentric positions, from which the required information could be reconstructed (cf. Sect. 4.5). For the sources with reference number 5, 7, 11, 15, and 16 in the last column of Table 1 , this is how the listed barycentric positions $\alpha(t)$ and $\delta(t)$ were derived, with their uncertainties, while the parallaxes and proper motions were taken from the cited references.

For Mira variables and red supergiants, the VLBI observations locate several maser spots in the very extended ( $\sim 100$ mas) circumstellar envelopes. When a kinematic model is employed for the relative motions of the spots caused by expansion and rotation of the envelope, it is often possible to infer the position of the geometrical centre of the star (e.g. Zhang et al. 2012; Nakagawa et al. 2014). The VLBI positions of these objects given in Table 1 are not accurate enough to contribute significantly to the determination of the rotation parameters, but the systemic proper motions may contribute to the spin. For reference number 14, the positions in Table 1 were taken from SIM$\mathrm{BAD}$ and are not used in the solutions except to compute the trigonometric factors in Eq. (14).

For some older observation series, in particular, Lestrade et al. (1999, reference number 3), the original results have been updated using more accurate calibrator (quasar) positions from the ICRF3 catalogue $^{2}$, resulting in some considerable improvements. An example is Cyg X-1 (HD 226868), where the positional uncertainties at 1991.25 were reduced from $\left(\sigma_{\alpha *}, \sigma_{\delta}\right)=(1.24,1.75)$ mas, as given in Lestrade et al. (1999), to the $(0.308,0.368)$ mas of Table 1.

In Eq. (17) the VLBI data are compared with the Gaia data propagated to the epoch $(t)$ of the VLBI data. This was done using the formulae in Appendix A, which requires knowledge of the radial motion of the source in order to take perspective effects into account. When available, radial velocities were taken from SIMBAD (Wenger et al. 2000); otherwise, a value of zero was used. The VLBI data were assumed to be uncorrelated, that is, all matrices $\boldsymbol{V}_{i}$ were taken as diagonal. For the present application, the most critical correlation is that between position and proper motion, which should normally be small if $t$ is close to the mean epoch of the VLBI measurements.

\footnotetext{
2 https://WWW.iers.org/IERS/EN/DataProducts/ICRF/ ICRF3/icrf3.html, using the S/X data. In this process the effect of the Galactic acceleration on the quasar positions was neglected.
} 
Table 1. Astrometric parameters determined by VLBI for the radio sources included in the analysis.

\begin{tabular}{|c|c|c|c|c|c|c|c|c|c|}
\hline Name & Type & $\begin{array}{c}v_{\mathrm{r}} \\
\left(\mathrm{km} \mathrm{s}^{-1}\right)\end{array}$ & $\begin{array}{c}\text { Epoch } t \\
\text { (Julian year) }\end{array}$ & $\begin{array}{c}\alpha(t) \\
(\operatorname{deg} \pm \text { mas }) \\
\end{array}$ & $\begin{array}{c}\delta(t) \\
(\operatorname{deg} \pm \text { mas }) \\
\end{array}$ & $\begin{array}{l}\varpi(t) \\
\text { (mas) }\end{array}$ & $\begin{array}{c}\mu_{\alpha *}(t) \\
\left(\operatorname{mas} \mathrm{yr}^{-1}\right)\end{array}$ & $\begin{array}{c}\mu_{\delta}(t) \\
\left(\operatorname{mas}^{-1}\right)\end{array}$ & Ref. \\
\hline SY Scl & Mira & 24.0 & 2006.8282 & $1.901028361 \pm 15.10$ & $-25.494452258 \pm 7.700$ & $0.750 \pm 0.030$ & $5.570 \pm 0.040$ & $-7.320 \pm 0.120$ & 1 \\
\hline S Per & RedSG & -39.7 & 2000.8884 & $35.715460833 \pm 7.818$ & $58.586512222 \pm 8.000$ & $0.413 \pm 0.017$ & $-0.490 \pm 0.230$ & $-1.190 \pm 0.200$ & 2 \\
\hline LS I +61303 & HXB & -41.4 & 1992.0000 & $40.131935007 \pm 0.291$ & $61.229332410 \pm 0.573$ & $0.260 \pm 0.610$ & $0.967 \pm 0.260$ & $-1.210 \pm 0.320$ & 3 \\
\hline \multirow[t]{2}{*}{ UX Ari } & RSCVn & 50.7 & 1999.9863 & $51.647432750 \pm 1.200$ & $28.715076528 \pm 0.800$ & $19.900 \pm 0.500$ & $44.960 \pm 0.130$ & $-102.330 \pm 0.090$ & 4 \\
\hline & & & 1991.2471 & $51.647339076 \pm 0.331$ & $28.715340363 \pm 0.377$ & $19.370 \pm 0.390$ & $41.617 \pm 0.186$ & $-104.010 \pm 0.200$ & 3 \\
\hline HD 22468 & RSCVn & -15.3 & 1992.0000 & $54.197113376 \pm 0.406$ & $0.588121131 \pm 0.401$ & $33.880 \pm 0.470$ & $-31.588 \pm 0.330$ & $-161.690 \pm 0.310$ & 3 \\
\hline V1271 Tau & RSCVn & 5.5 & 2013.0000 & $55.951464140 \pm 0.110$ & $25.004223050 \pm 0.171$ & $7.418 \pm 0.025$ & $19.860 \pm 0.050$ & $-45.410 \pm 0.160$ & 5 \\
\hline V811 Tau & BYDra & 11.3 & 2013.0000 & $56.338353995 \pm 0.118$ & $23.727308022 \pm 0.198$ & $7.223 \pm 0.057$ & $19.470 \pm 0.110$ & $-44.390 \pm 0.270$ & 5 \\
\hline \multirow[t]{2}{*}{ HD 283447} & T Tau & 16.0 & 2006.9700 & $63.553843529 \pm 0.132$ & $28.203380745 \pm 0.097$ & $7.700 \pm 0.190$ & $17.092 \pm 0.077$ & & 6 \\
\hline & & & 1993.8809 & $63.553831265 \pm 0.312$ & $28.203455247 \pm 0.470$ & $6.740 \pm 0.250$ & $0.423 \pm 0.291$ & $-23.250 \pm 0.280$ & 3 \\
\hline V410 Tau & T Tau & 19.9 & 2015 & 64.6296612 & 28.4543779 & $7.751 \pm 0$. & & & 7 \\
\hline V1023 Tau & $\mathrm{T}$ Tau & 12.7 & 2005 & 64.6959683 & 28.3353831 & $7.530 \pm 0.030$ & $4.300 \pm 0$ & \pm 0.300 & 8 \\
\hline \multirow[t]{2}{*}{ HD 283572} & $\mathrm{~T} \mathrm{Tau}$ & 14.2 & 2005.3 & 65.4952167 & $28.301769800 \pm 0.050$ & $7.780 \pm 0$ & & & 8 \\
\hline & & & 2005.3600 & $65.495216803 \pm 0.032$ & $28.301769751 \pm 0.035$ & $7.841 \pm 0.057$ & $9.023 \pm 0.061$ & $-26.445 \pm 0.077$ & 7 \\
\hline T Tau & T Tau & 23.9 & 2004.6231 & $65.497604438 \pm 0.028$ & $19.534921017 \pm 0.400$ & $6.820 \pm 0.030$ & & $-1.180 \pm 0.050$ & 9 \\
\hline HD 283641 & T Tau & 16.2 & 201 & 66.204409 & 26.7194801 & $6.285 \pm$ & 10.9 & -16.7 & 7 \\
\hline V111 & RSCVn & & & & & $11.881 \pm$ & & & 7 \\
\hline HD 282630 & T Tau & 13.6 & 2016.5800 & $73.904063653 \pm 0.067$ & $30.298527621 \pm 0.066$ & $7.061 \pm 0.125$ & $3.897 \pm 0.113$ & $-24.210 \pm 0.132$ & 7 \\
\hline T Lep & Mira & -4.0 & 2005.6427 & $76.211833879 \pm 30.18$ & $-21.904579275 \pm 13.15$ & $3.060 \pm 0.040$ & $14.600 \pm 0.500$ & $-35.430 \pm 0.790$ & 10 \\
\hline V1961 & Orion_V* & 30.1 & & 027 & .028 & $2.533 \pm$ & & & 11 \\
\hline Brun 334 & PMS & 21.3 & & 023 & $21 \pm 0.048$ & $2.591 \pm$ & & & 11 \\
\hline V1321 Ori & Orion_V* & 17.6 & 2015.1800 & $83.767921447 \pm 0.075$ & $-5.136841216 \pm 0.182$ & $2.509 \pm 0.044$ & 0.06 & $6.950 \pm 0.160$ & 11 \\
\hline MT Ori & Orion_V* & & 2015.1800 & $83.824801264 \pm 0.030$ & $-5.379288056 \pm 0.062$ & $2.646 \pm 0.041$ & $3.820 \pm 0.100$ & $1.600 \pm 0.170$ & 11 \\
\hline V1046 Ori & $\mathrm{SB}$ & 29.5 & & & & $2.643 \pm 0$ & & & 11 \\
\hline HD 3 & Star & 10.8 & & 84.062621 & $74 \pm 0.096$ & $2.536=$ & 1.32 & -0.56 & 11 \\
\hline TYC 5 & Star & & & \pm 0.058 & -8.12088430 & $2.348 \pm 0$. & & -0.51 & 11 \\
\hline HD 290862 & Star & & 2015.2100 & $86.680772978 \pm 0.491$ & $0.076677687 \pm 0.643$ & $2.197 \pm 0.545$ & $0.350 \pm 0.270$ & $0.830 \pm 0.830$ & 11 \\
\hline [SSC75] M 7811 & Star & & 2015.2100 & $86.688907482 \pm 0.043$ & $0.044527387 \pm 0.085$ & $2.547 \pm 0.034$ & $0.010 \pm 0.100$ & $-0.490 \pm 0.080$ & 11 \\
\hline VY CMa & RedSG & 60.8 & & $3 \pm 10.00$ & \pm 10.00 & 80 & & & 12 \\
\hline $\mathrm{SCrt}$ & & 32.0 & & 1.800 & \pm 13.30 & $2.330 \pm 0$ & -3 . & -5.41 & 13 \\
\hline BH CVn & RSCVn & 6.4 & 1993.1088 & $203.698997828 \pm 0.373$ & $37.182433334 \pm 0.455$ & $22.210 \pm 0.450$ & $85.496 \pm 0.131$ & $-9.220 \pm 0.160$ & 3 \\
\hline $\mathrm{S} \mathrm{CrB}$ & Mira & -5.1 & 2000.0000 & 230.349817010 & 31.367381400 & $2.360 \pm 0.230$ & $-9.060 \pm 0.230$ & $-12.520 \pm 0.290$ & 14 \\
\hline$\sigma^{2} \mathrm{CrB}$ & HPM & -14.8 & 1990.0014 & $243.671114605 \pm 0.104$ & $33.858853887 \pm 0.124$ & $43.930 \pm 0.100$ & $-267.048 \pm 0.037$ & $-86.660 \pm 0.050$ & 3 \\
\hline U Her & & -26.1 & & 246.44779 & & $3.740 \pm 0$ & $-14.980 \pm 0.290$ & -9.23 & 14 \\
\hline Haro 1-6 & T Tau & & 200 & $246.512566654 \pm 0.399$ & $-24.393446456 \pm 0.356$ & $7.385 \pm 0.234$ & $-19.630 \pm$ & -26.92 & 15 \\
\hline DoAr 51 & T Tau & & 2014.7500 & $248.049128879 \pm 0.337$ & $-24.672768034 \pm 0.336$ & $6.983 \pm 0.050$ & $-4.800 \pm 0.080$ & $-23.110 \pm 0.110$ & 15 \\
\hline W 40 IRS 5 & Star & & 2014.9500 & $277.811761649 \pm 0.065$ & $-2.063932732 \pm 0.119$ & $2.302 \pm 0.063$ & $0.186 \pm 0.053$ & $-6.726 \pm 0.121$ & 16 \\
\hline RR Aql & Mira & 11.0 & 2000.0000 & 299.400248870 & -1.886482960 & $1.580 \pm 0.400$ & $-25.110 \pm 0.740$ & $-49.820 \pm 0.540$ & 14 \\
\hline \multirow[t]{2}{*}{ Cyg X-1 } & HXB & -2.7 & & $303145 \pm 0.500$ & $35.201590284 \pm 0.500$ & $0.547 \pm 0$ & & & 17 \\
\hline & & & 1991.2498 & $299.590326964 \pm 0.308$ & $35.201622230 \pm 0.368$ & & $-3.787 \pm 0.172$ & -6.2 & 3 \\
\hline HD 199178 & RVS & -26.6 & 1993.7933 & $313.473486712 \pm 0.332$ & $44.386412565 \pm 0.397$ & $8.590 \pm 0.330$ & $26.595 \pm 0.407$ & $-1.240 \pm 0.430$ & 3 \\
\hline SS Cyg & DwarfNova & -62.0 & 2011.5661 & $325.678846337 \pm 0.065$ & $43.586181392 \pm 0.070$ & $8.800 \pm 0.120$ & $112.420 \pm 0.070$ & $33.380 \pm 0.070$ & 18 \\
\hline AR Lac & RSCVn & -33.8 & 1992.4353 & $332.170232910 \pm 0.274$ & $45.742153188 \pm 0.361$ & $23.970 \pm 0.370$ & $-52.080 \pm 0.126$ & $47.030 \pm 0.190$ & 3 \\
\hline \multirow[t]{2}{*}{ IM Peg } & RSCVn & -14.4 & 2005.0869 & $343.259410883 \pm 0.400$ & $16.841155569 \pm 0.390$ & $10.370 \pm 0.074$ & $-20.833 \pm 0.090$ & $-27.267 \pm 0.095$ & 19 \\
\hline & & & & $343.259484359 \pm 0.360$ & $16.841247861 \pm 0.392$ & $10.280 \pm 0.620$ & $-20.587 \pm 0.459$ & $-27.530 \pm 0.400$ & 3 \\
\hline PZ Cas & RedSG & -51.4 & 2006.2998 & $356.013673333 \pm 2.836$ & $61.789496389 \pm 3.000$ & $0.356 \pm 0.026$ & $-3.700 \pm 0.200$ & $-2.000 \pm 0.300$ & 20 \\
\hline
\end{tabular}

Notes. Name, type, and radial velocity $\left(v_{\mathrm{r}}\right)$ are taken from SIMBAD, except for the names Cyg X-1 (for HD 226868) and $\sigma^{2} \mathrm{CrB}$ (for sig CrB A). An ellipsis (..) means the same data as in the line above. Positions $\alpha(t), \delta(t)$ are barycentric and refer to the epochs in the fourth column. Uncertainties (in mas and mas $\mathrm{yr}^{-1}$ ) are given after the $\pm \operatorname{sign}$; for $\alpha$ they are $\sigma_{\alpha *}=\sigma_{\alpha} \cos \delta$. Positional data without uncertainties were not used in the solutions.

References. (1) Nyu et al. (2011); (2) Asaki et al. (2010); (3) Lestrade et al. (1999); (4) Peterson et al. (2011); (5) Melis et al. (2014); (6) Torres et al. (2012); (7) Galli et al. (2018); (8) Torres et al. (2007); (9) Loinard et al. (2007); (10) Nakagawa et al. (2014); (11) Kounkel et al. (2017); (12) Zhang et al. (2012); (13) Nakagawa et al. (2008); (14) Vlemmings \& van Langevelde (2007); (15) Ortiz-León et al. (2017a); (16) Ortiz-León et al. (2017b); (17) Reid et al. (2011); (18) Miller-Jones et al. (2013); (19) Bartel et al. (2015); (20) Kusuno et al. (2013).

\subsection{Gaia data}

Gaia DR2 identifiers for the optical counterparts of the radio sources are given in Table 2. For most sources they were taken directly from SIMBAD, but in a few cases (HD 22468, T Tau, VY CMa, and AR Lac), they had to be derived by cross-matching the radio positions with the Gaia positions. Relevant optical data were retrieved from the Gaia Archive ${ }^{3}$ and the full covariance matrices $\boldsymbol{C}_{i}$ computed from the formal uncertainties and

\footnotetext{
3 https://gea.esac.esa.int/archive/
}

correlation coefficients. As these data are readily available on-line, they are not reproduced in Table 2, with the exception of the $G$ magnitude and the re-normalised unit weight error (RUWE). The latter, computed from Archive data as described in Lindegren (2018), is a goodness-of-fit measure (formally the square root of the reduced chi-square of the astrometric solution) that should be around 1.0 for an astrometrically well-behaved source. RUWE $\gtrsim 1.4$ could indicate an astrometric binary, a (partially) resolved binary or multiple star, or an otherwise problematic source. The remaining columns in Table 2 are explained below. 
Table 2. Gaia DR2 matches and solution statistics for the radio sources in Table 1.

\begin{tabular}{|c|c|c|c|c|c|c|c|c|}
\hline Name & Gaia DR2 identifier & $\begin{array}{c}G \\
(\mathrm{mag}) \\
\end{array}$ & RUWE & $\begin{array}{c}E_{i} \\
\left(\mathrm{mas}^{-2}\right)\end{array}$ & $\begin{array}{c}\Omega_{i} \\
\left(\mathrm{mas}^{-2} \mathrm{yr}^{2}\right)\end{array}$ & $n_{i}$ & $Q_{i} / n_{i}$ & $\begin{array}{c}\text { Accepted } \\
(0 / 1) \\
\end{array}$ \\
\hline SY Scl & 2335529621301024128 & 10.09 & 1.28 & 0.0 & 20.2 & 5 & 2.459 & 1 \\
\hline S Per & 459101393719884800 & 7.80 & 1.27 & 0.0 & 16.6 & 5 & 4.336 & 1 \\
\hline LS I +61 303 & 465645515129855872 & 10.39 & 0.92 & 1.4 & 755.1 & 5 & 9.440 & 1 \\
\hline UX Ari & 118986060277836160 & 6.33 & 6.33 & 0.7 & 12.1 & 10 & 381.455 & 0 \\
\hline HD 22468 & 3263936692671872384 & 5.60 & 1.01 & 0.3 & 143.7 & 5 & 3.346 & 1 \\
\hline V1271 Tau & 69876712724339456 & 11.44 & 1.15 & 54.6 & 197.4 & 5 & 0.309 & 1 \\
\hline V811 Tau & 65190048708380160 & 12.09 & 1.08 & 42.1 & 240.4 & 5 & 3.213 & 1 \\
\hline HD 283447 & 163184366130809984 & 9.98 & 3.99 & 21.8 & 23.1 & 10 & 5179.761 & 0 \\
\hline V410 Tau & 164518589131083136 & 10.32 & 0.98 & 1156.3 & 339.1 & 5 & 4.977 & 1 \\
\hline V1023 Tau & 164513538249595136 & 11.65 & 3.27 & 3.9 & 27.1 & 5 & 1235.511 & 0 \\
\hline HD 283572 & 164536250037820160 & 8.80 & 1.02 & 10.2 & 273.7 & 10 & 1.228 & 1 \\
\hline $\mathrm{T}$ Tau & 48192969034959232 & 9.63 & 1.69 & 12.0 & 165.6 & 5 & 163141.331 & 0 \\
\hline HD 283641 & 152104381299305600 & 10.80 & 1.62 & 210.6 & 110.1 & 5 & 4.112 & 1 \\
\hline V1110 Tau & 147940663906992512 & 9.96 & 0.90 & 147.6 & 203.3 & 5 & 85.411 & 0 \\
\hline HD 282630 & 156900622818205312 & 10.33 & 1.02 & 255.1 & 260.1 & 5 & 73.922 & 0 \\
\hline T Lep & 2962625495403737600 & 6.44 & 1.39 & 0.0 & 5.3 & 5 & 70.453 & 0 \\
\hline V1961 Ori & 3209424108758593408 & 11.61 & 1.09 & 605.3 & 201.2 & 5 & 0.554 & 1 \\
\hline Brun 334 & 3017270879709003520 & 10.82 & 1.10 & 699.3 & 169.8 & 5 & 4.013 & 1 \\
\hline V1321 Ori & 3209531650444835840 & 10.13 & 0.96 & 176.8 & 72.1 & 5 & 0.720 & 1 \\
\hline MT Ori & 3017364127743299328 & 11.32 & 1.16 & 479.0 & 97.3 & 5 & 64.307 & 0 \\
\hline V1046 Ori & 3209634905754969856 & 6.53 & 2.21 & 100.1 & 14.8 & 5 & 34.425 & 0 \\
\hline HD 37150 & 3017294622299833216 & 6.51 & 0.98 & 294.4 & 95.9 & 5 & 47.427 & 0 \\
\hline TYC 5346-538-1 & 3015742318025842944 & 10.65 & 0.93 & 205.8 & 59.6 & 5 & 12.414 & 1 \\
\hline HD 290862 & 3219148872492984192 & 10.18 & 1.65 & 6.4 & 13.6 & 5 & 16.074 & 1 \\
\hline [SSC75] M 7811 & 3219148185299243776 & 12.51 & 1.10 & 371.8 & 167.7 & 5 & 606.173 & 0 \\
\hline VY CMa & 5616197646838713728 & 7.17 & 17.20 & 0.0 & 0.7 & 5 & 43.240 & 0 \\
\hline $\mathrm{S} \mathrm{Crt}$ & 3595101382979440256 & 6.42 & 1.40 & 0.2 & 27.5 & 5 & 30.878 & 0 \\
\hline $\mathrm{BH} \mathrm{CVn}$ & 1475118788534734592 & 4.73 & 0.85 & 0.3 & 77.4 & 5 & 2.045 & 1 \\
\hline $\mathrm{S} \mathrm{CrB}$ & 1277100833181122816 & 7.40 & 1.70 & 0.0 & 8.4 & 3 & 5.873 & 1 \\
\hline$\sigma^{2} \mathrm{CrB}$ & 1328866562170960512 & 5.41 & 1.14 & 1.9 & 158.2 & 5 & 229.860 & 0 \\
\hline U Her & 1200834239913483392 & 7.04 & 1.35 & 0.0 & 14.3 & 3 & 5.248 & 1 \\
\hline Haro 1-6 & 6049142032584969088 & 12.24 & 0.96 & 2.6 & 91.7 & 5 & 1.715 & 1 \\
\hline DoAr 51 & 6047570826172040960 & 12.52 & 6.43 & 10.2 & 3.2 & 5 & 1.261 & 1 \\
\hline W 40 IRS 5 & 4270236599432306560 & 12.13 & 2.98 & 46.5 & 15.4 & 5 & 9156.399 & 0 \\
\hline RR Aql & 4234448531043979520 & 8.18 & 1.55 & 0.0 & 4.0 & 3 & 12.668 & 1 \\
\hline Cyg X-1 & 2059383668236814720 & 8.52 & 0.98 & 6.3 & 736.5 & 10 & 1.261 & 1 \\
\hline HD 199178 & 2162964329341318656 & 7.01 & 0.88 & 1.0 & 463.1 & 5 & 0.546 & 1 \\
\hline SS Cyg & 1972957892448494592 & 11.69 & 1.42 & 32.9 & 181.1 & 5 & 1.023 & 1 \\
\hline AR Lac & 1962909425622345728 & 5.89 & 0.82 & 1.9 & 933.1 & 5 & 0.884 & 1 \\
\hline IM Peg & 2829193299742131328 & 5.66 & 1.43 & 4.0 & 115.3 & 10 & 1.269 & 1 \\
\hline PZ Cas & 2012859963999694848 & 6.64 & 1.06 & 0.2 & 40.9 & 5 & 3.400 & 1 \\
\hline
\end{tabular}

Notes. $G$ is the mean magnitude in the Gaia photometric band. RUWE is the re-normalised unit weight error (Lindegren 2018) of the astrometric solution for the source in Gaia DR2. $E_{i}$ and $\Omega_{i}$ are the formal weights potentially contributed by the source to the estimation of $\boldsymbol{\varepsilon}(T)$ and $\omega$, computed using Eq. (29), and $n_{i}$ the number of VLBI data items on the source. The penultimate column is the discrepancy measure from Eq. (28), and the last column lists whether the source was accepted (1) or discarded (0) in the baseline solution.

It is known that the parallaxes in Gaia DR2 are systematically too small by a few tens of microarcseconds (Arenou et al. 2018). The zero-point is estimated to be about -0.03 mas for the faint quasars (Lindegren et al. 2018), but there is strong evidence that the bright stars of interest here have a more negative zero-point, around -0.05 mas (e.g. Riess et al. 2018; Zinn et al. 2018; Schönrich et al. 2019). This is broadly confirmed by the VLBI data: the median parallax difference for the 41 radio stars in Table 1 is $\varpi_{\mathrm{DR} 2}-\varpi_{\mathrm{VLBI}}=-0.076 \pm 0.025$ mas. In the solutions reported below, the Gaia DR2 parallaxes of all the radio stars have been increased by 0.05 mas to take the zero-point error into account. Because parallax and the other astrometric parameters in the Gaia data are correlated, this changes the estimated rotation parameters, but only by small amounts: about 0.010 mas in

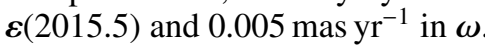

\subsection{Results}

Direct application of the solution method in Sect. 2.3 to the full sample of 41 sources gives a very poor fit as measured by the loss function: $Q \simeq 920915$ for $n \equiv \sum_{i} n_{i}=224$ degrees of freedom, or a reduced chi-square of $Q / n \simeq 4111$. This solution also gives unrealistically high values for the spin parameters, with $|\hat{\omega}| \simeq 1.5 \mathrm{mas} \mathrm{yr}^{-1}$. Inspection of the discrepancy measure 
$Q_{i} / n_{i}$ of the individual sources shows that T Tau has by far the highest value $Q_{i} / n_{i} \simeq 159819$, followed by W 40 IRS 5 at $Q_{i} / n_{i} \simeq 8681$, and so on. Removing T Tau from the sample and re-computing the solution and discrepancy measures gives $Q \simeq$ 113037 for $n=\sum_{i} n_{i}=219$ degrees of freedom $(Q / n \simeq 516)$. In this solution the source with the largest discrepancy measure is W 40 IRS 5 at $Q_{i} / n_{i} \simeq 8714$. Removing this source as well and iterating the procedure until all sources but one have been removed gives a series of solutions with $k=0,1, \ldots$ rejected sources. The evolution of $\max \left(Q_{i} / n_{i}\right)$ and $Q / n$ as functions of $k$ is shown in the left panels of Fig. 3, with the corresponding orientation and spin parameters in the right panels.

Errors in the rotation parameters caused by non-linear source motions and other model deficiencies are generally reduced as more outliers are removed, while the statistical (formal) uncertainties increase because fewer sources contribute to the solution. The optimum solution is a compromise between the opposite tendencies and may be found somewhere along the sequence of solutions described above. The rather smooth progression of the discrepancy measures in Fig. $3 \mathrm{a}$ and $\mathrm{b}$ gives no clear indication of the optimum $k$, except that it is probably in the range from 7 (removing the most obvious outliers) to $\simeq 30$ (after which $Q / n<1)$. The spin parameters in Fig. $3 \mathrm{~d}$ show an abrupt change with the removal of HD 282630 at $k=11$, after which the spin solution is relatively stable in the $Y$ and $Z$ components, but less so in $X$. The orientation parameters in Fig. 3c show a similar behaviour. Any $k$ in the range from 13 to 35 in fact gives fairly consistent solutions at least in $Y$ and $Z$. For the subsequent discussion, we take somewhat arbitrarily the solution at $k=15$ as the baseline. This solution has $Q / n=3.55$ with $n=139$ degrees of freedom, and the most discrepant source is HD 290863 with $Q_{i} / n_{i} \simeq 16.074$.

The accepted and rejected sources and their individual discrepancy measures for the baseline solution are listed in Table 2. The rotation parameters are given in Table 3 under entry A, including the formal uncertainties calculated from the inverse normal matrix. The correlation matrix (for $T=\mathrm{J} 2015.5$ ) is

$$
\begin{aligned}
\operatorname{corr} & {\left[\varepsilon_{X}(T), \varepsilon_{Y}(T), \varepsilon_{Z}(T), \omega_{X}, \omega_{Y}, \omega_{Z}\right] } \\
& =\left[\begin{array}{llllll}
+1.000 & +0.517 & +0.204 & +0.136 & +0.066 & +0.120 \\
+0.517 & +1.000 & +0.206 & +0.099 & +0.131 & +0.105 \\
+0.204 & +0.206 & +1.000 & +0.091 & +0.035 & +0.133 \\
+0.136 & +0.099 & +0.091 & +1.000 & -0.028 & +0.405 \\
+0.066 & +0.131 & +0.035 & -0.028 & +1.000 & -0.090 \\
+0.120 & +0.105 & +0.133 & +0.405 & -0.090 & +1.000
\end{array}\right]
\end{aligned}
$$

Given the reduced chi-square of the solution, $Q / n \simeq 3.55$, it is likely that the formal uncertainties underestimate the actual errors. More realistic estimates may be obtained by bootstrap resampling of the 26 accepted sources, yielding the uncertainties in the "Adopted" entry of Table 3.

From the original sample of $m=41$ sources, $k=15$ were thus removed to obtain the baseline subset of $m-k=26$ sources. It is not obvious that the process of successively removing the most discrepant source leads to the optimum subset in the sense that no other subset of the same size has a smaller $Q / n$. It is conceivable that a different procedure, for instance, starting from a smaller subset and adding the best-fitting sources (outward selection; Ben-Gal 2010), would lead to a different result. While it is impractical to test all $\left(\begin{array}{l}41 \\ 26\end{array}\right) \simeq 6 \times 10^{10}$ possible combinations, an exhaustive search of the $\left(\begin{array}{l}33 \\ 26\end{array}\right)=4272048$ different subsets of size 26 drawn from the 33 sources with the smallest discrepancy measure did not uncover any more favourable subset. Figure 4 displays $Q / n$ versus the spin components for these solutions. Many of them are quite different from the baseline solution in terms of the spin components, but invariably, their $Q / n$ is then also significantly higher. This makes it credible that the spin parameters of the adopted solution are statistically significant and not the chance result of a particular combination of data for a few sources.

The solution gives improved estimates of the astrometric parameters of the sources, obtained by solving (21) for each $i$. These results are not tabulated, as they are practically the same as obtained from a weighted average of the VLBI and Gaia data after correcting the latter for the frame rotation and parallax zero-point. For example, the joint estimate of the parallax of Cyg X-1 is $\hat{\varpi}=0.505 \pm 0.025$ mas, very close to the weighted mean of the VLBI value, $0.547 \pm 0.041$ mas, and the Gaia DR2 value after correction for the zero-point, $0.472 \pm 0.032$ mas.

\subsection{Alternative solutions}

The classical way to determine $\omega$ is to solve the overdetermined system of Eqs. (8) and (9) using only the proper motion differences. In this process it is natural to assign a weight to each equation that is inversely proportional to the combined variances of the VLBI and Gaia proper motions. An equivalently weighted least-squares solution is obtained with the formalism of Sect. 2.3 simply by deleting in $f_{i}$ all the VLBI data items that are not proper motions. The resulting normal equations, Eq. (22), are of course singular for the orientation parameters, but the lowerright $3 \times 3$ part of the equations gives the desired solution for $\omega$. Applying this procedure to the baseline subset of 26 sources gives the result shown as solution $B$ in Table 3 . The spin parameters are reasonably close to those of the baseline solution (A), testifying to the good internal consistency of the (accepted) data. It is more interesting, however, that the formal uncertainties are significantly higher in solution B than in A: the uncertainty is a factor two higher for the $Y$ component.

It thus appears that the positional VLBI data are at least as valuable as the proper motion data when estimating the spin, at least for the spread of VLBI epochs considered here. A direct test of this hypothesis is to make the complementary solution to what is described above, that is, deleting all proper motion items in $\boldsymbol{f}_{i}$. The result is shown as solution $\mathrm{C}$ in Table 3 . In this case, both the orientation and spin parameters are determined, and the spin is actually more precise (in terms of formal uncertainties) than in solution B where the proper motions (only) are used, although not as precise as in A. It can be noted that only 23 of the 26 sources contribute to solution $\mathrm{C}$ because no positional VLBI data are provided for S CrB, U Her, and RR Aql, although these sources belong to the baseline subset.

Figure 4 of Lindegren et al. (2018) suggests that the transition from the faint to the bright reference frame in Gaia DR2 does not occur abruptly at $G=13$ but gradually from $G \simeq 13$ to $\simeq 11 \mathrm{mag}$. Several of the sources in Table 1 have magnitudes in the transition interval and may therefore not contribute fully to the determination of the rotation parameters. In solution $D$ the model of the Gaia data in (11) is modified so that the applied rotation is $\boldsymbol{x}$ multiplied by the magnitude-dependent function

$\phi(G)= \begin{cases}1 & \text { if } G \leq 11, \\ (13-G) / 2 & \text { if } 11<G \leq 13, \\ 0 & \text { if } G>13\end{cases}$ 

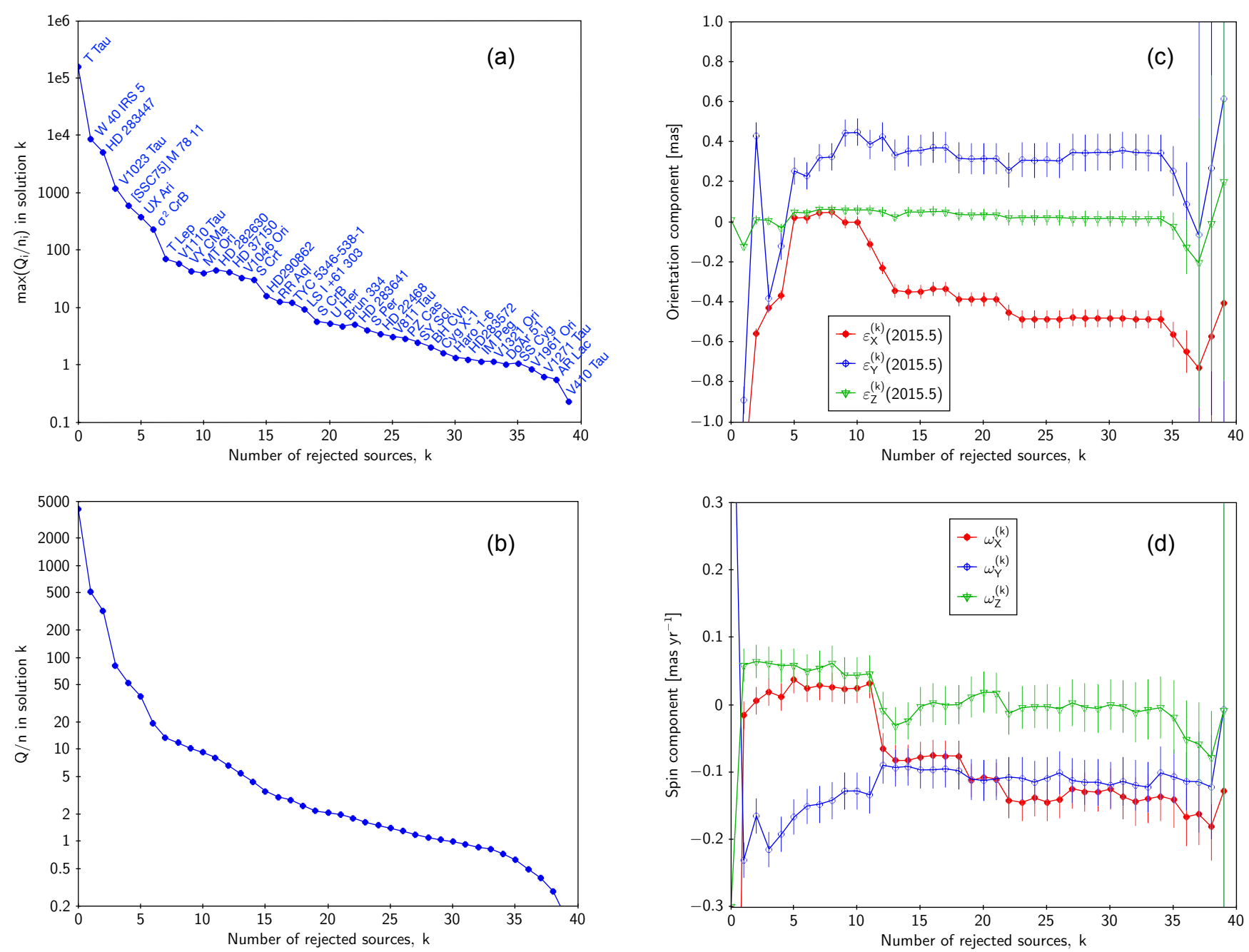

Fig. 3. Evolution of discrepancy measures and rotation parameters in a series of solutions where the $k$ most discrepant sources have been removed. $a$ : discrepancy measure $Q_{i} / n_{i}$ for the most discrepant source in the solution. Points are labelled with the name of the source. $b$ : total discrepancy measure $Q / n$ as a function of the number of rejected sources. $c$ : estimated orientation parameters. $d$ : spin parameters as functions of the number of rejected sources. Error bars are formal $\pm 1 \sigma$ uncertainties from the inverse normal matrix, i.e. not adjusted depending on the goodness-of-fit.

Table 3. Summary of the different solutions for the orientation and spin parameters.

\begin{tabular}{|c|c|c|c|c|c|c|}
\hline \multirow[t]{2}{*}{ Solution } & \multicolumn{3}{|c|}{ Orientation at $T=\mathrm{J} 2015.5$ (mas) } & \multicolumn{3}{|c|}{$\operatorname{Spin}\left(\mathrm{mas} \mathrm{yr}^{-1}\right)$} \\
\hline & $\varepsilon_{X}(T)$ & $\varepsilon_{Y}(T)$ & $\varepsilon_{Z}(T)$ & $\omega_{X}$ & $\omega_{Y}$ & $\omega_{Z}$ \\
\hline A & $-0.347 \pm 0.032$ & $+0.358 \pm 0.074$ & $+0.050 \pm 0.026$ & $-0.077 \pm 0.023$ & $-0.096 \pm 0.027$ & $-0.002 \pm 0.028$ \\
\hline $\mathrm{B}$ & - & - & - & $-0.050 \pm 0.036$ & $-0.139 \pm 0.055$ & $+0.002 \pm 0.038$ \\
\hline $\mathrm{C}$ & $-0.361 \pm 0.036$ & $+0.443 \pm 0.085$ & $+0.052 \pm 0.033$ & $-0.087 \pm 0.028$ & $-0.100 \pm 0.028$ & $-0.013 \pm 0.035$ \\
\hline $\mathrm{D}$ & $-0.358 \pm 0.034$ & $+0.403 \pm 0.079$ & $+0.059 \pm 0.028$ & $-0.059 \pm 0.025$ & $-0.112 \pm 0.029$ & $+0.010 \pm 0.030$ \\
\hline Adopted & $-0.347 \pm 0.137$ & $+0.358 \pm 0.245$ & $+0.050 \pm 0.045$ & $-0.077 \pm 0.051$ & $-0.096 \pm 0.042$ & $-0.002 \pm 0.036$ \\
\hline
\end{tabular}

Notes. The table gives the estimated orientation and spin components for different solutions, with their uncertainties (after \pm ). All solutions use the same subset of 26 sources, marked as accepted in Table 2. Solution A is the baseline solution described in Sect. 3.3. Solutions B-D are the alternative solutions described in Sect. 3.4: B using only proper motions, C using only positions, and D using the same data as A and the magnitude dependent model in Eq. (31). The "Adopted" solution is identical to A, but the uncertainties are estimated by bootstrap resampling of the 26 sources (while for A-D the uncertainties are formal values computed from the inverse normal matrix).

Unfortunately, this does not improve the overall fit significantly ( $Q=492.80$ in D against 493.14 in A), and the discrepancy measure actually increases for four of the six accepted sources in the magnitude range $11-13$. Thus the VLBI data do not clearly support the magnitude-dependent model, although it cannot be ruled out either.

\subsection{Weights of the individual sources}

Table 2 includes the statistics $E_{i}$ and $\Omega_{i}$ from Eq. (29), indicating the potential weights of the sources in the orientation and spin solutions. The sources that contribute most weight to the spin solution (largest $\Omega_{i}$ ) are AR Lac, LS I +61 303, Cyg X-1, 

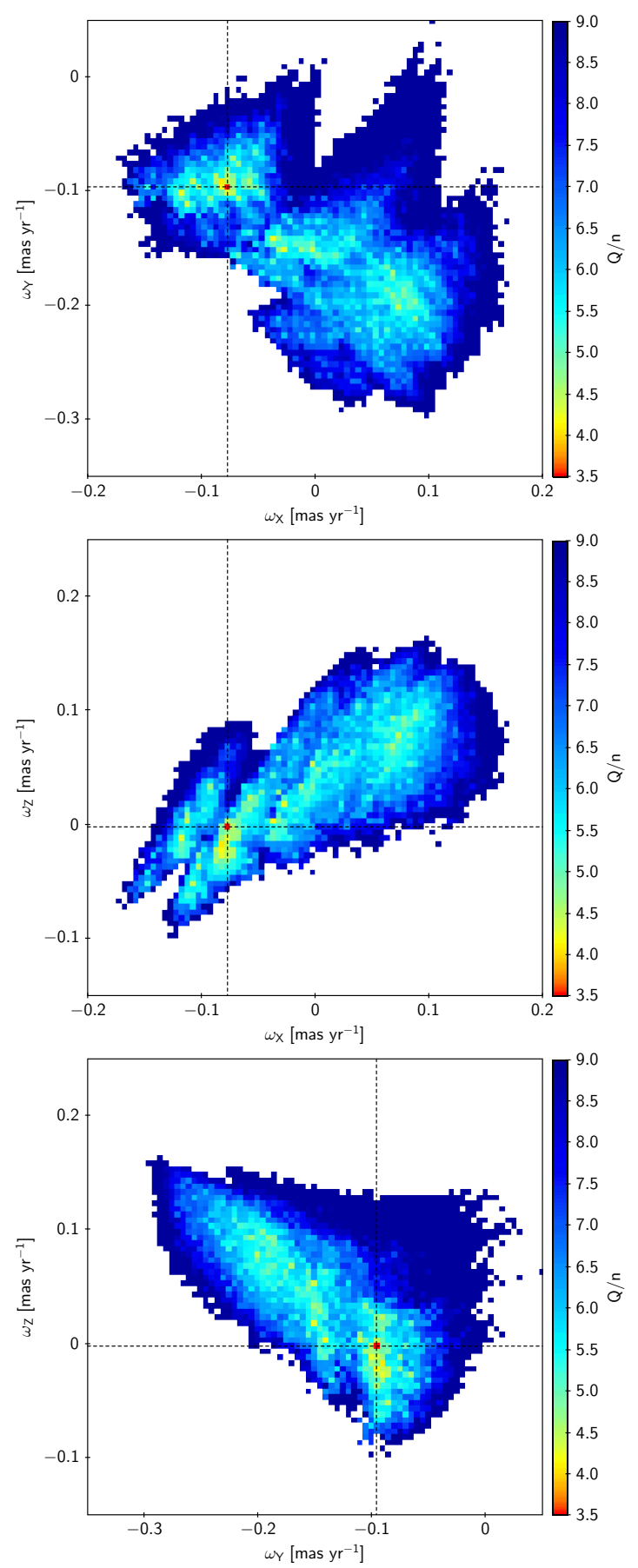

Fig. 4. Sensitivity of the solution to the selection of sources. The plots show the spin parameters and associated $Q / n$ values for about four million solutions using different subsets of the sources as described in the text. The colour shows the smallest $Q / n$ in each bin. The dashed lines mark the spin parameters for the baseline solution A with $Q / n \simeq 3.55$.

HD 199178, and V410 Tau; all of them have $\Omega_{i}>300 \mathrm{mas}^{-2} \mathrm{yr}^{2}$ and all are included in the baseline subset. The first three and HD 199178 mainly contribute by virtue of their relatively small positional uncertainties $(\simeq 0.3$ mas) at a very early epoch, $t \simeq$ 1992. IM Peg, one of the best-observed radio stars (Bartel et al. 2015), has a much smaller weight in this analysis because its uncertainties in Gaia DR2 are relatively large.

The main contributions to the determination of the orientation (largest $E_{i}$ ) come from young stellar objects in the Taurus and Orion regions that are observed as part of the GOBELINS

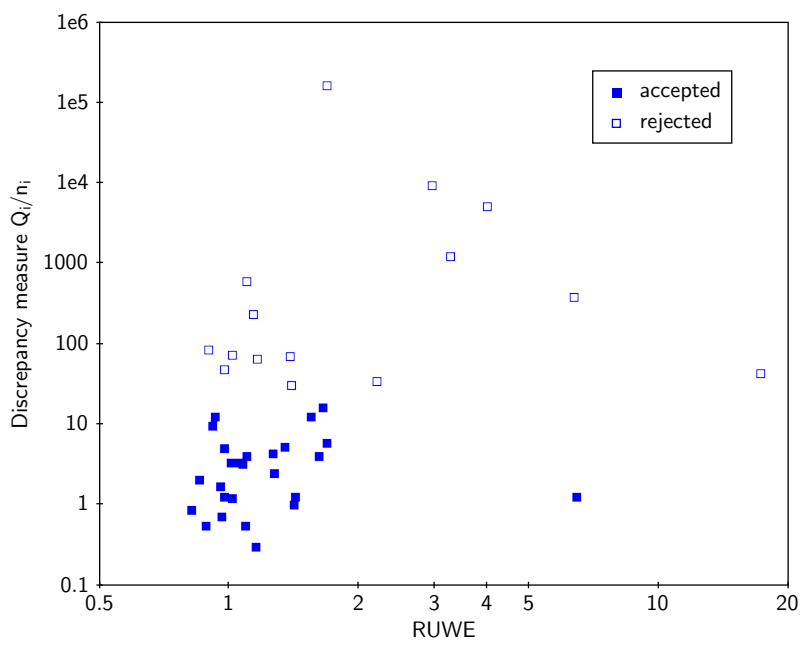

Fig. 5. Discrepancy measures of the sources in solution A against the RUWE in the Gaia DR2 astrometry.

survey (Galli et al. 2018; Kounkel et al. 2017), through their high precision and proximity in time with the Gaia DR2 epoch. The strong concentration of these sources in a small part of the sky, approximately in the $+Y$ direction, is responsible for the relatively large uncertainty of the $Y$ component of $\boldsymbol{\varepsilon}(2015.5)$ in Table 3.

\section{Discussion}

\subsection{Estimated rotation parameters}

The result in Table 3 yields a spin for the Gaia DR2 bright reference frame of $\omega=(-0.077,-0.096,-0.002)$ mas $^{-1} r^{-1}$, significant at the $2 \sigma$ level in the $Y$ component. Figure $3 \mathrm{~d}$ indeed suggests that $\omega_{Y}$ is quite firmly fixed around $-0.1 \mathrm{mas} \mathrm{yr}^{-1}$, while the results in $Z$ and (especially) $X$ are less certain.

The orientation parameters $\boldsymbol{\varepsilon}(2015.5)$ are less well determined, and especially the $Y$ component has a large uncertainty that is due to the unfavourable celestial distribution of the more recent VLBI observations included in the analysis (Sect. 3.5). Still, an orientation error of about 0.5 mas is indicated at the $2 \sigma$ level in the $X$ and $Y$ components.

As mentioned in the Introduction, the non-zero spin of the bright reference frame of Gaia DR2 is seen also in a comparison with proper motions of HIPPARCos stars calculated from the position differences between Gaia DR2 and the HIPPARCOS catalogue, divided by the $\sim 24 \mathrm{yr}$ epoch difference. This comparison was made by Brandt (2018) in the course of constructing The HIPPARCOS-Gaia Catalog of Accelerations (HGCA). For 115663 HIPPARCos stars the HGCA gives three essentially independent sets of proper motions, namely (i) as measured by HIPPARCOS around epoch 1991.25, (ii) as measured by Gaia (in DR2) around epoch 2015.5, and (iii) as calculated from the HIPPARCOS-Gaia position differences divided by the epoch difference. The HGCA is intended for orbit fitting and for identifying candidate stars with substellar or dark companions, and to this end, Brandt (2018) made a careful cross-calibration of the three sets of proper motions in order to eliminate any systematic offsets, in particular the global rotations. For the rotation between sets (ii) and (iii) the result as given in his Table 1 is $\omega=(-0.081,-0.113,-0.038)$ mas $\mathrm{yr}^{-1}$. Brandt used the same sign convention for this vector as in this paper, so that his result can be directly compared with our Table 3 . The agreement is 
very good for all the solutions in Table 3; for the adopted solution, none of the component differences exceeds one standard deviation.

The comparison with Brandt (2018) rests on the assumption that the proper motions calculated from the HIPPARCOS-Gaia position differences are absolute, that is, that they are expressed in a reference frame that is non-rotating with respect to the ICRS $^{4}$. The validity of this assumption depends on the quality of the positional reference frame of HIPPARCOS at epoch 1991.25 and of the positional reference frame for the same stars in Gaia DR2 at epoch 2015.5. If both sets of positions are aligned with the ICRS at their respective epochs, the proper motions calculated from the position differences must clearly be non-rotating with respect to the ICRS. In The HIPPARCOS and Tycho Catalogues (ESA 1997, Vol. 3, Sect. 18.7) the rms orientation error of the HIPPARCos reference frame at epoch 1991.25 is given as 0.6 mas per axis. For the bright DR2 frame at epoch 2015.5 we may conclude from Cols. $2-4$ in Table 3 that the rms error is probably smaller than 0.4 mas per axis. This gives a total rms

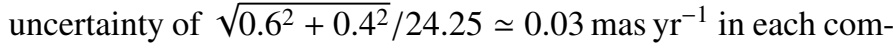
ponent of $\omega$.

The old HIPPARCos positions thus provide an independent estimate of the spin, in good agreement with the results from the VLBI observations, and of comparable or even better accuracy. However, because its uncertainty is dominated by the unknown orientation errors of the HIPPARCOS reference frame, which will not improve, the method will be of limited value for the validation of future Gaia data releases. In contrast, the VLBI method, as discussed in Sect. 4.4, has great potential for future improvement.

The origin of the non-zero rotation parameters for the bright reference frame of Gaia DR2 is a deficiency in the specific instrument calibration model used for the DR2 astrometric solution. The relevance of this deficiency for the reference frame was not recognised at the time when the DR2 data were prepared and validated, and although its effect on the bright sources was noted (e.g. Fig. 4 in Lindegren et al. 2018), no explanation of its origin was offered. This is now understood, and the calibration model is being improved with a view to avoid a similar error in future data releases (see Appendix B).

\subsection{Use of positional VLBI data}

A comparison of the formal uncertainties of $\omega$ in solutions A, B, and $\mathrm{C}$ (Table 3 ) demonstrates the advantage of including positional VLBI data in a joint solution for the orientation and spin parameters. Not only do the positional data, as already noted, improve the spin solution ( $\mathrm{A}$ is better than $\mathrm{B}$ for $\omega$ ), but the inclusion of proper motion data also improves the orientation parameters ( $\mathrm{A}$ is better than $\mathrm{C}$ for $\boldsymbol{\varepsilon}$ ).

The value of positional VLBI data for the spin is especially noteworthy because it means that even single-epoch VLBI astrometry can be incorporated in the solution. This will contribute to the spin determination, especially if the data are taken at an epoch that is well separated from the Gaia epoch. This is important to keep in mind, both for the inclusion of old, possibly unpublished VLBI measurements (Sect. 4.5) and for the

\footnotetext{
4 Brandt (2018) adopted the (bright) reference frame of Gaia DR2 for the HGCA, therefore the published proper motions of type (iii), called pmra_hg and pmdec_hg in his Table 5, are not absolute in our sense. To place them on the ICRS, the applied cross-calibration corrections must be subtracted, i.e. use pmra_hg-crosscal_pmra_hg and pmdec_hgcrosscal_pmdec_hg.
}

scheduling of future VLBI sessions specifically for the reference frame (Sect. 4.4). The latter need not be constrained by considerations of parallax factor and temporal spread of the measurements for the proper motions.

\subsection{Binarity and source structure}

About half of the radio sources in Table 1 are known to be binaries or members of double or multiple systems. Interacting pairs with periods from a day to tens of days include the RS CVn systems and high-mass X-ray binaries, which usually provide good fits to the single-star model unless they are perturbed by a more distant component, as is the case for $\sigma^{2} \mathrm{CrB}$. Binaries with periods of years to hundreds of years are more problematic unless a complete orbit can be determined. Orbits have been determined for some radio stars, but as the corresponding binary data from Gaia are not yet available, they are not included in this analysis. For other objects, the VLBI observations have detected the curvature of a long-period orbit by means of acceleration terms; this is the case for example for UX Ari, HD 283447, and T Tau, all of which obtain large discrepancy measures in the present analysis (Fig. 3a).

The radio stars included in the present analysis have not been a priori screened for known or anticipated problems with multiplicity and source structure. Apart from known binaries, several Mira variables and red supergiants have therefore been included, although they are far from ideal targets because of the issues described in Sect. 3.1. It is very likely that the optical Gaia observations are also severely affected by the extended and complex atmospheres (Chiavassa et al. 2011). This type of radio star should probably be avoided entirely for the reference frame.

A statistic that could be used for screening the sources is the RUWE given in Table 2. The RUWE measures how well the different Gaia observations that were made over a few years agree with the five-parameter single-star model. It is therefore mainly sensitive to the presence of companions at separations from a few milliarcsecond to about one arcsecond, and a relevant indicator of potentially problematic sources of that particular kind. It is not sensitive to non-linear motions, if the deviation from linear is small over the few years of the Gaia observations. A large discrepancy measure $Q_{i} / n_{i}$ also indicates a problematic source, but of a rather different kind. In contrast to the RUWE, it is sensitive to radio-optical offsets, and it is also more sensitive to longperiod perturbations if the VLBI and Gaia measurements are made at very different epochs. Figure 5 shows a weak positive correlation between the two statistics, which is to be expected because their different regimes of sensitivity overlap. However, it is clear that RUWE alone is not sufficient to find the best candidate targets for determination of the frame rotation parameters.

Surprisingly, one source (DoAr 51) has a very large RUWE $=6.43$, but nevertheless gives a good fit in the present solution $\left(Q_{i} / n_{i}=1.261\right)$. This object is a triple system consisting of an equal-mass pair with a period of about 8 years and a separation of approximately 56 mas at 2015.5, and a fainter tertiary component at a separation of about 790 mas (Schaefer et al. 2018). This triple configuration could well explain the high RUWE obtained with Gaia. The VLBI observations detect both components of the close pair, and the data in Table 1 refer to their centre of mass (Ortiz-León et al. 2017a). The nearcoincidence of the VLBI and Gaia epochs, together with the near-coincidence of the optical photocentre with the centre of mass of the close pair and the rather large uncertainty of the Gaia proper motion $\left(\sim 1\right.$ mas $\left.\mathrm{yr}^{-1}\right)$, could explain why the discrepancy measure is not higher. With $\Omega_{i}=3.2 \mathrm{mas}^{-2} \mathrm{yr}^{2}$, this 
Table 4. Formal improvement in the determination of orientation and spin parameters expected from the addition of new positional VLBI data for the 26 sources in the baseline subsample.

\begin{tabular}{lccc}
\hline \hline Gaia data & $\begin{array}{c}\text { Added VLBI data } \\
\left(\sigma_{\text {pos }}=0.1 \text { mas }\right)\end{array}$ & $\begin{array}{c}\sigma[\boldsymbol{\varepsilon}(T)] \\
(\mu \mathrm{as})\end{array}$ & $\begin{array}{c}\sigma[\omega] \\
\left(\mu \mathrm{as} \mathrm{yr}^{-1}\right)\end{array}$ \\
\hline DR2 $(T=2015.5)$ & None & 48.9 & 26.1 \\
-"- & $t=2020$ & 25.3 & 21.8 \\
-"- & $t=2025$ & 26.7 & 21.3 \\
-"- & $t=2030$ & 27.8 & 21.2 \\
\hline 5yr $(T=2017.1)$ & None & 49.6 & 9.6 \\
-"- & $t=2020$ & 22.1 & 7.0 \\
-"- & $t=2025$ & 22.9 & 5.8 \\
-"- & $t=2030$ & 23.7 & 5.3 \\
\hline 10yr $(T=2019.6)$ & None & 57.0 & 6.9 \\
-"- & $t=2020$ & 22.5 & 4.6 \\
-"- & $t=2025$ & 21.0 & 3.6 \\
-"- & $t=2030$ & 21.7 & 2.9 \\
\hline
\end{tabular}

Notes. $T$ is the reference epoch used in the solution; $t$ is the epoch of the added VLBI observations. The last two columns give the formal uncertainties in $\varepsilon(T)$ and $\omega$, calculated as the quadratic means of the uncertainties in the $X, Y$, and $Z$ components. The top entry (DR2 without added VLBI data) corresponds to solution A in Table 3.

object contributes less to the determination of $\omega$ than any of the other accepted sources in Table 2. DoAr 51 is a good example of an object for which an extended model along the lines in Sect. 2.4 could drastically increase the usefulness of the data.

\subsection{Precision of future solutions}

The bright reference frame in future releases of Gaia data should ideally be validated at a level compatible with the expected errors of the proper motions, which may be as small as a few $\mu a \mathrm{yr}^{-1}$. This will require a much better accuracy in the spin parameters than is achieved in the present analysis. Clearly, the uncertainties can be reduced by including VLBI data for more radio stars, and/or using improved data for the sources already considered. As discussed in Sect. 4.2, the epoch of the added data is an important factor. If dedicated VLBI measurements are contemplated for this purpose, different scenarios can be envisaged concerning the number, distribution, and epochs of the planned observations, and it is of great interest to predict the accuracy that can be achieved in various cases. This can be done by applying the algorithm in Sect. 2.3 to simulated data sets, using the left-hand side of Eq. (22) to compute the formal precisions.

A major uncertainty in any such prediction is the extent to which binarity, source structure, and radio-optical offsets will limit the achievable accuracy. One extreme scenario is that these effects already dominate the error budget in the current analysis. Support for this could be drawn from the fact that $Q_{i} / n_{i}>1$ for nearly all the accepted sources in Table 2 . In this scenario it will not help much to add more and better VLBI data for the radio stars already considered, and the safest way to improve the solution may be to increase the number of sources, $m$, and rely on the statistical improvement by $m^{-1 / 2}$.

Realistically, however, the prospects are not as bleak as outlined above. Better screening of the sample, modelling of orbital motions and offsets, etc., will surely improve the results, and this process will be helped by the addition of new data both from VLBI and Gaia. The scenario at the other extreme is that such improvements will allow us to reach the formal uncertainties computed from the least-squares equations. This (optimistic) assumption is the basis for the predictions in Table 4 .

The first entry in Table 4 represents the baseline solution of the present analysis (A in Table 3). For brevity, the formal uncertainties of the six rotation parameters are condensed here into two numbers, one for the orientation and one for the spin. For the next three entries, it is assumed that new VLBI observations of the same 26 accepted sources are obtained at the specified epoch $t$, with a precision of 0.1 mas in each coordinate. Performing the analysis as in Sect. 2.3 with the same Gaia DR2 data, we obtain the formal uncertainties in the last two columns. Remarkably, while the orientation (at $T=2015.5$ ) is better determined with the added data, the improvement is small in the spin and practically independent of the epoch of the added VLBI measurements. This shows that the formal uncertainties of the spin in solution A are limited by the accuracy of the proper motions in Gaia DR2 rather than by the VLBI data. The slight increase in $\sigma[\boldsymbol{\varepsilon}(T)]$ with $t$ arises because the effective mean epoch of the (old plus new) VLBI measurements moves away from the reference epoch $T$.

The middle four entries in Table 4 show predictions when Gaia data are used based on the nominal mission length $L=$ $5 \mathrm{yr}$. It is assumed that the uncertainties of the Gaia astrometry improve as $L^{-1 / 2}$ for the positions and parallaxes, and as $L^{-3 / 2}$ for the proper motions (taking $L=1.8 \mathrm{yr}$ for DR2). The covariance matrices $\boldsymbol{C}_{i}$ in (11) are simply scaled by the corresponding factors, leaving the correlations unchanged from DR2. The orientation parameters now refer to the corresponding reference epoch of the Gaia observations, $T=2017.1^{5}$. Consistent with the previous finding that the DR2 precision is the main limitation in solution A, the improved Gaia data drastically reduce the formal uncertainty of the spin parameters even without any additional VLBI data. Adding new data for the 26 stars improves the determination of the spin still further, especially if the new measurements are at a late epoch. The orientation parameters, on the other hand, are not much improved. Their uncertainties are basically limited by the VLBI position errors, typically of the order of 0.1 mas, and the small number of sources: $0.1 / \sqrt{26} \simeq$ 0.02 mas.

The last four entries in Table 4 are for an extended Gaia mission covering a full decade of observations $(L=10 \mathrm{yr})$. Here the spin parameters receive another boost in precision, while there is almost no improvement in the orientation parameters. The $\sigma[\varepsilon(T)]$ has a shallow minimum at $t \simeq 2024$ because the effective mean epoch of the VLBI measurements is then close to the assumed reference epoch, $T=2019.6$.

Taking into account that the two extreme scenarios considered above are probably to some extent true, we conclude from this crude assessment that a combination of actions should be taken to ensure that a robust and accurate estimate of the rotation parameters can be derived at the time when the final Gaia results become available. These actions should include compiling and recalibrating past VLBI measurements, as well as securing new observations of both old targets and as many new as possible.

\subsection{A plea to VLBI observers}

Most of the observational VLBI programmes used in this study address problems in Galactic or stellar astrophysics, and are

\footnotetext{
5 The reference epochs of future data releases are not known at the present time. The values in the table are assumed for the purpose of this study and correspond to the approximate intervals 2014.6-2016.4 (DR2), 2014.6-2019.6, and 2014.6-2024.6.
} 
primarily concerned with the parallaxes and proper motions of the radio stars, or of their orbits, surface structures, and similar. Consequently, many of the publications do not provide the (barycentric) positions that the authors must have derived along with the parallaxes and proper motions by fitting an astrometric model to their positional measurements. This is not a problem as long as the authors provide the individual measurements, and their times, on which the fit was based. For this reason, as mentioned in Sect. 3.1, several of the barycentric positions in Table 1 were derived by the present author by fitting the standard model (Appendix A) to the published VLBI measurements. Although this procedure is not without advantages (see below), it is of course simpler if the full solution is provided by the observers.

In this context, it is useful to stress the unique historical value of positional data. In contrast to the parallaxes, for example, which can be re-determined at a future time, positional measurements can never be repeated, and for a number of applications their value only increases with time. A plea to observers making high-precision VLBI astrometry is therefore that they publish the full result of their astrometric fits, including the barycentric position and corresponding epoch.

It is nevertheless good practice to publish the individual position measurements for future uses as well. This allows alternative models, which may include acceleration terms or orbital parameters for example, to be fitted in combination with other data. The general method described in Sect. 2.3 is readily adapted to the use of individual VLBI measurements, and this has the advantage that otherwise neglected correlations are fully taken into account.

Relative astrometry using phase-referencing techniques are converted into absolute coordinates using an assumed position in the ICRS of the reference source (calibrator), usually a quasar. To first order, small errors in the calibrator position directly transfer to the measured coordinates of the target (Reid $\&$ Honma 2014). This gives a constant offset of no consequence when the parallax and proper motion of the target are fitted to the data, but it might be important for the present application. It is customary to specify the calibrators used in phasereferencing observations, and sometimes also their assumed positions. Knowing the identities and adopted positions of the calibrators is indeed highly desirable because it allows the target positions to be corrected when improved calibrator positions become available (cf. Sect. 3.1).

\section{Conclusions}

This paper provides a rigorous mathematical framework for estimating the orientation and spin of the Gaia reference frame, in which the Gaia data are optimally combined with VLBI measurements of bright radio sources. The simultaneous estimation of the orientation $(\varepsilon)$ and spin $(\omega)$ is essential for achieving the best accuracy. The method takes full advantage of past and future single-epoch VLBI measurements of Gaia sources for the determination of the spin. Independent estimates of their proper motions from VLBI can be incorporated in the solution, but are not required by the method.

Applied to published VLBI data for a sample of 41 bright ( $G \leq 13 \mathrm{mag}$ ) radio sources, the method indicates that the bright reference frame of Gaia DR2 rotates by about 0.1 mas yr$^{-1}$ relative to the faint quasar frame. The solution retains 26 of the investigated radio sources, while 15 are rejected based on a statistical discrepancy measure sensitive to binarity and source structure. The adopted non-zero result in Table 3 is significant at the $2 \sigma$ level. The orientation error of $\simeq 0.5$ mas of the bright reference frame with respect to ICRS also appears to be significant.

This determination of the spin is consistent with independent estimates obtained from a comparison of the extrapolated Gaia DR2 positions with the HIPPARCos catalogue at epoch J1991.25 (Lindegren et al. 2018; Brandt 2018).

The accuracy of the present study is limited by the relatively small number of radio stars included, by the uncertainties of the Gaia DR2 proper motions, and by issues related to the astrophysical nature of the sources.

The origin of the spin of the bright reference frame of Gaia DR2 is understood, and measures have been taken to to avoid this problem in future data releases (see Appendix B). Nevertheless, it is important that the consistency of future reference frames can be validated across the full range of magnitudes, and the present method offers such a possibility for the bright stars. As many as possible of the already existing VLBI measurements of radio stars should be used for this purpose, but it is very desirable to complement this by re-observing many of them in the coming years, and if possible, add new targets to the list for improved robustness. The use and recalibration of old, possibly unpublished data should be pursued. In this context, the unique historical value of positional VLBI measurements needs to be stressed. As argued in Sect. 4.5, observers should ensure that relevant intermediate data and meta-information are preserved for optimal future uses of their data.

Acknowledgements. I wish to thank S. Klioner for many stimulating discussions on this and related topics, and for a critical reading of the first draft. I also thank the anonymous referee and A. Bombrun, U. Bastian, M. Biermann, J. Castañeda, M. Davidson, C. Fabricius, E. Gerlach, J. Hernández, D. Hobbs, R. Le Poole, P. McMillan, M. Ramos-Lerate, and N. Rowell for valuable comments and advice. Support from the Swedish National Space Board is gratefully acknowledged. This work has made use of data from the European Space Agency (ESA) mission Gaia (https://www. cosmos.esa.int/gaia), processed by the Gaia Data Processing and Analysis Consortium (DPAC, https://www. cosmos.esa.int/web/gaia/dpac/consortium). Funding for the DPAC has been provided by national institutions, in particular the institutions participating in the Gaia Multilateral Agreement. The work has also made use of the SIMBAD database, operated at CDS, Strasbourg, France (Wenger et al. 2000). Calculations were made using MATLAB by The MathWorks, Inc. Diagrams were produced using the astronomy-oriented data handling and visualisation software TOPCAT (Taylor 2005).

\section{References}

Arenou, F., Luri, X., Babusiaux, C., et al. 2018, A\&A, 616, A17

Arias, E. F., Charlot, P., Feissel, M., \& Lestrade, J. F. 1995, A\&A, 303, 604

Arias, E. F., Feissel, M., \& Lestrade, J. F. 1988, A\&A, 199, 357

Asaki, Y., Deguchi, S., Imai, H., et al. 2010, ApJ, 721, 267

Bartel, N., Bietenholz, M. F., Lebach, D. E., et al. 2015, Class. Quant. Grav., 32, 224021

Beasley, A. J., \& Conway, J. E. 1995, in Very Long Baseline Interferometry and the VLBA, eds. J. A. Zensus, P. J. Diamond, \& P. J. Napier, ASP Conf. Ser., 82,327

Ben-Gal, I. 2010, in Data Mining and Knowledge Discovery Handbook, eds. O. Maimon, \& L. Rokach, 2nd ed. (Boston, MA: Springer), 117

Björck, Å. 1996, Numerical Methods for Least Squares Problems (Philadelphia: Society for Industrial and Applied Mathematics)

Bobylev, V. V. 2015, Astron. Lett., 41, 156

Brandt, T. D. 2018, ApJS, 239, 31

Brosche, P., Ducourant, C., Galas, R., Geffert, M., \& Karafistan, A. 1991, A\&A, 245, 669

Brunthaler, A., Reid, M. J., Menten, K. M., et al. 2011, Astron. Nachr., 332, 461 Butkevich, A. G., \& Lindegren, L. 2014, A\&A, 570, A62

Chiavassa, A., Pasquato, E., Jorissen, A., et al. 2011, A\&A, 528, A120

Crowley, C., Kohley, R., Hambly, N. C., et al. 2016, A\&A, 595, A6

de Vegt, C., \& Ebner, H. 1974, MNRAS, 167, 169

ESA 1997, The Hipparcos and Tycho Catalogues (Noordwijk: ESA), ESA SP1200

Evans, D. W., Riello, M., De Angeli, F., et al. 2018, A\&A, 616, A4 
Fedorov, P. N., Akhmetov, V. S., \& Bobylev, V. V. 2011, MNRAS, 416, 403

Fomalont, E. B. 2012, in Astrometry for Astrophysics: Methods, Models, and Applications, ed. W. Van Altena, 175

Fricke, W. 1977, Veroeffentlichungen des Astronomischen Rechen-Instituts Heidelberg, 28, 52

Froeschle, M., \& Kovalevsky, J. 1982, A\&A, 116, 89

Gaia Collaboration (Prusti, T., et al.) 2016, A\&A, 595, A1

Gaia Collaboration (Mignard, F., et al.) 2018a, A\&A, 616, A14

Gaia Collaboration (Brown, A. G. A., et al.) 2018b, A\&A, 616, A1

Galli, P. A. B., Loinard, L., Ortiz-Léon, G. N., et al. 2018, ApJ, 859, 33

Honma, M. 2013, in New Trends in Radio Astronomy in the ALMA Era: The 30th Anniversary of Nobeyama Radio Observatory, eds. R. Kawabe, N. Kuno, \& S. Yamamoto, ASP Conf. Ser., 476, 81

Jacobs, C., Charlot, P., Arias, E. F., et al. 2018, in 42nd COSPAR Scientific Assembly, COSPAR Meeting, 42, B2.1-31-18

Klioner, S. A. 2003, AJ, 125, 1580

Kounkel, M., Hartmann, L., Loinard, L., et al. 2017, ApJ, 834, 142

Kovalevsky, J. 2003, A\&A, 404, 743

Kovalevsky, J., Lindegren, L., Perryman, M. A. C., et al. 1997, A\&A, 323, 620

Kusuno, K., Asaki, Y., Imai, H., \& Oyama, T. 2013, ApJ, 774, 107

Lestrade, J.-F., Rogers, A. E. E., Whitney, A. R., et al. 1990, AJ, 99, 1663

Lestrade, J. F., Jones, D. L., Preston, R. A., et al. 1995, A\&A, 304, 182

Lestrade, J.-F., Preston, R. A., Jones, D. L., et al. 1999, A\&A, 344, 1014

Lindegren, L. 2018, Gaia Technical Note GAIA-C3-TN-LU-LL-124, http : // www.rssd.esa.int/doc_fetch.php?id=3757412

Lindegren, L., \& Kovalevsky, J. 1995, A\&A, 304, 189

Lindegren, L., Lammers, U., Hobbs, D., et al. 2012, A\&A, 538, A78

Lindegren, L., Lammers, U., Bastian, U., et al. 2016, A\&A, 595, A4

Lindegren, L., Hernández, J., Bombrun, A., et al. 2018, A\&A, 616, A2

Loinard, L., Torres, R. M., Mioduszewski, A. J., et al. 2007, ApJ, 671, 546

Ma, C., Arias, E. F., Eubanks, T. M., et al. 1998, AJ, 116, 516

Matthews, L. D. 2019, PASP, 131, 016001

Melis, C., Reid, M. J., Mioduszewski, A. J., Stauffer, J. R., \& Bower, G. C. 2014 Science, 345, 1029

Metz, M., \& Geffert, M. 2004, A\&A, 413, 771
Miller-Jones, J. C. A., Sivakoff, G. R., Knigge, C., et al. 2013, Science, 340, 950 Murray, C. A. 1983, Vectorial Astrometry (Bristol: Adam Hilger) Nakagawa, A., Tsushima, M., Ando, K., et al. 2008, PASJ, 60, 1013 Nakagawa, A., Omodaka, T., Handa, T., et al. 2014, PASJ, 66, 101 Nyu, D., Nakagawa, A., Matsui, M., et al. 2011, PASJ, 63, 63 Ortiz-León, G. N., Loinard, L., Kounkel, M. A., et al. 2017a, ApJ, 834, 141 Ortiz-León, G. N., Dzib, S. A., Kounkel, M. A., et al. 2017b, ApJ, 834, 143 Ortiz-León, G. N., Loinard, L., Dzib, S. A., et al. 2018, ApJ, 865, 73

Peterson, W. M., Mutel, R. L., Lestrade, J.-F., Güdel, M., \& Goss, W. M. 2011, ApJ, 737, 104

Reid, M. J., \& Honma, M. 2014, ARA\&A, 52, 339

Reid, M. J., McClintock, J. E., Narayan, R., et al. 2011, ApJ, 742, 83

Riess, A. G., Casertano, S., Yuan, W., et al. 2018, ApJ, 861, 126

Rioja, M., \& Dodson, R. 2011, AJ, 141, 114

Schaefer, G. H., Prato, L., \& Simon, M. 2018, AJ, 155, 109

Schlesinger, F. 1917, AJ, 30, 137

Schönrich, R., McMillan, P., \& Eyer, L. 2019, MNRAS, 487, 3568

Sovers, O. J., Fanselow, J. L., \& Jacobs, C. S. 1998, Rev. Mod. Phys., 70, 1393

Taylor, M. B. 2005, in Astronomical Data Analysis Software and Systems XIV, eds. P. Shopbell, M. Britton, \& R. Ebert, ASP Conf. Ser., 347, 29

Torres, R. M., Loinard, L., Mioduszewski, A. J., \& Rodríguez, L. F. 2007, ApJ, 671,1813

Torres, R. M., Loinard, L., Mioduszewski, A. J., et al. 2012, ApJ, 747, 18

Vlemmings, W. H. T., \& van Langevelde, H. J. 2007, A\&A, 472, 547

Walter, H. G., \& Sovers, O. J. 2000, Astrometry of Fundamental Catalogues: The Evolution from Optical to Radio Reference Frames (Berlin, Heidelberg: Springer-Verlag)

Wenger, M., Ochsenbein, F., Egret, D., et al. 2000, A\&AS, 143, 9

Wolf, H. 1978, Proceedings of the Second International Symposium on Problems Related to the Redefinition of North American Geodetic Networks (Washington, DC: US Department of Commerce), 319

Xu, S., Zhang, B., Reid, M. J., Zheng, X., \& Wang, G. 2019, ApJ, 875, 114

Zhang, B., Reid, M. J., Menten, K. M., \& Zheng, X. W. 2012, ApJ, 744, 23

Zhu, Z. 2000, PASP, 112, 1103

Zinn, J. C., Pinsonneault, M. H., Huber, D., \& Stello, D. 2018, ApJ, 878, A136 


\section{Appendix A: Standard model of stellar motion}

The propagation of stellar astrometric data in time is normally based on a set of approximations, assumed to be valid for the observed motion of a single star, or the centre of mass of a binary or multiple system, over a limited interval of decades to centuries. Referred to in The HIPPARCos and Tycho Catalogues (ESA 1997, Vol. 1, Sect. 1.2.8) as "The Standard Model of Stellar Motion", these approximations have been adopted as the basis for astrometric reductions at least since the days of Schlesinger (1917) and are still used for the analysis of Gaia data (Lindegren et al. 2012). Among the most important explicit or implicit assumptions of the model are (i) that the source moves with constant velocity vector relative to the Solar System barycentre, (ii) that light-time effects beyond the Solar System can be ignored, and (iii) that aberration effects caused by the curvature of the Galactic orbits can be ignored. This is not the place to discuss the validity of these assumptions ${ }^{6}$, but because the model, formally represented by the function $\boldsymbol{F}_{i}\left(\boldsymbol{y}_{i}\right)$ in (15), is central to the paper, it may be useful to summarise the main steps of the calculation. The Barycentric Celestial Reference System (BCRS) is used throughout, with times expressed in seconds or Julian years of barycentric coordinate time (TCB), and distances in $\mathrm{km}$ or au.

\section{A.1. Propagation of the astrometric parameters}

We first consider the propagation of the astrometric parameters from epoch $T$ to $t$. Let $\alpha, \delta, \varpi, \mu_{\alpha *}, \mu_{\delta}$, and $v_{\mathrm{r}}$ be the astrometric parameters and radial velocity at the original epoch $T$, and $\alpha(t)$, etc. their values at epoch $t$. The first step is to compute the barycentric unit vector towards the source at time $T$,

$\boldsymbol{r}=\left[\begin{array}{c}\cos \alpha \cos \delta \\ \sin \alpha \cos \delta \\ \sin \delta\end{array}\right]$

and the unit vectors in the directions of increasing $\alpha$ and $\delta$,

$\boldsymbol{p}=\left[\begin{array}{c}-\sin \alpha \\ \cos \alpha \\ 0\end{array}\right], \quad \boldsymbol{q}=\left[\begin{array}{c}-\cos \alpha \sin \delta \\ -\sin \alpha \sin \delta \\ \cos \delta\end{array}\right]$

Next we compute

$\boldsymbol{m}=\mu_{\alpha *} \boldsymbol{p}+\mu_{\delta} \boldsymbol{q}+\left(v_{\mathrm{r}} \varpi / A\right) \boldsymbol{r}$,

where

$A=\frac{149597870.7 \mathrm{~km}}{365.25 \times 86400 \mathrm{~s} \mathrm{yr}^{-1}} \simeq 4.740470464 \mathrm{~km} \mathrm{yr} \mathrm{s}^{-1}$

is the astronomical unit. The vectors $\boldsymbol{r}$ and $\boldsymbol{m}$ are such that for constant space velocity, the barycentric vector to the source at time $t$ is proportional to

$\boldsymbol{s}(t)=\boldsymbol{r}+(t-T) \boldsymbol{m}$.

The astrometric parameters at epoch $t$ are thus recovered from $\boldsymbol{s}(t)$ and $\boldsymbol{m}$ after rescaling and performing the inverse operations

\footnotetext{
6 Assumptions (i) and (ii) are discussed by Butkevich \& Lindegren (2014) and (iii) is discussed by Kovalevsky (2003). For the practical definition of the astrometric parameters in a relativistic framework, see Klioner (2003).
}

of (A.1)-(A.3):

$$
\begin{aligned}
& \boldsymbol{r}(t)=|\boldsymbol{s}(t)|^{-1} \boldsymbol{s}(t), \quad \boldsymbol{m}(t)=|\boldsymbol{s}(t)|^{-1} \boldsymbol{m}, \\
& \alpha(t)=\operatorname{atan} 2\left(r_{Y}(t), r_{X}(t)\right), \\
& \delta(t)=\operatorname{atan} 2\left(r_{Z}(t), \sqrt{r_{X}(t)^{2}+r_{Y}(t)^{2}}\right), \\
& \varpi(t)=|\boldsymbol{s}(t)|^{-1} \varpi, \\
& \mu_{\alpha *}(t)=\boldsymbol{p}(t)^{\prime} \boldsymbol{m}(t), \\
& \mu_{\delta}(t)=\boldsymbol{q}(t)^{\prime} \boldsymbol{m}(t), \\
& v_{\mathrm{r}}(t)=\boldsymbol{r}(t)^{\prime} \boldsymbol{m}(t) A / \varpi(t),
\end{aligned}
$$

where

$$
\boldsymbol{p}(t)=\left[\begin{array}{c}
-\sin \alpha(t) \\
\cos \alpha(t) \\
0
\end{array}\right], \quad \boldsymbol{q}(t)=\left[\begin{array}{c}
-\cos \alpha(t) \sin \delta(t) \\
-\sin \alpha(t) \sin \delta(t) \\
\cos \delta(t)
\end{array}\right]
$$

are the updated vectors along $+\alpha$ and $+\delta$.

\section{A.2. Coordinate direction to the source}

We now turn to the calculation of the position of the source as obtained from a single VLBI measurement at time $t$. As opposed to the barycentric direction $\boldsymbol{r}(t)$ discussed above, we now require the topocentric direction from the observer towards the source. In VLBI astrometry, geometric delays are calculated from the source positions and station coordinates expressed in the BCRS frame and corrected for the gravitational delay caused by bodies in the Solar System (for details, see Sovers et al. 1998). The astrometric position measured by VLBI therefore corresponds to the geometric direction from the observer towards the target at the time of observation, unaffected by stellar aberration and gravitational deflection. This direction, also known as the coordinate direction (Murray 1983), is here denoted $\overline{\boldsymbol{u}}(t)$.

The modelling of $\overline{\boldsymbol{u}}(t)$ in terms of the astrometric parameters is simple because it only involves a shift of origin from the Solar System barycentre to the observer. With $\boldsymbol{b}(t)$ denoting the position of the observer at the time of observation, expressed as BCRS coordinates in au, we have

$\overline{\boldsymbol{u}}(t)=\left\langle\boldsymbol{s}\left(t_{\mathrm{B}}\right)-\varpi \boldsymbol{b}(t)\right\rangle$,

where angular brackets signify vector normalisation, $\langle\boldsymbol{a}\rangle=\boldsymbol{a} /|\boldsymbol{a}|$. One small complication in Eq. (A.14) is that the position of the source should be evaluated for the barycentric time $t_{\mathrm{B}}$ obtained by adding the Römer delay to the time of observation,

$t_{\mathrm{B}}=t+\boldsymbol{r}(t)^{\prime} \boldsymbol{b}(t) / c$,

where $c$ is the speed of light. For an observer on the Earth, the Römer delay is at most about $500 \mathrm{~s}$. Neglecting the delay produces an error equal to the proper motion of the source over this time interval, which could amount to 0.17 mas for Barnard's star. While the effect is thus negligible for most stars, it is always safer to take it into account. On the other hand, it is an acceptable approximation to use $\boldsymbol{r}$ instead of $\boldsymbol{r}(t)$ in (A.15). (For the position of the observer, $\boldsymbol{b}(t)$, we can normally take the centre of the Earth, which is readily available from standard ephemerides, although the diurnal parallax of the nearest star is as much as $32 \mu \mathrm{as})$. The celestial coordinates of the source are finally obtained from the $X, Y, Z$ components of $\overline{\boldsymbol{u}}(t)$ in analogy with Eqs. (A.7) and (A.8). 


\section{Appendix B: Origin of the rotation of the bright reference frame of Gaia DR2}

As mentioned in Sect. 4.1, the deviating reference frame for bright $(G \lesssim 13$ ) sources in Gaia DR2 is caused by a deficiency in the astrometric instrument calibration model used for DR2. Although a detailed discussion of the effect is beyond the scope of this paper, it may be useful to outline the basic mechanism and how the effect can be avoided in future data releases. Further details may be found in the documentation of forthcoming releases.

The astrometric calibration model used for Gaia DR2 is described in Sect. 3.3 of Lindegren et al. (2018). It contains several terms that depend on the "window class" (WC) of the individual CCD observation. Window classes WC0/1/2 are different schemes for sampling the pixels around a detected source (for details, see Sect. 3.3.5 in Gaia Collaboration 2016). The WC is decided by an on-board algorithm, based mainly on the $G$ magnitude of the source estimated as it enters the field of view. WC0 $(G \lesssim 13$ ) provides a small two-dimensional image of the source, from which both the along- and across-scan coordinates can be derived. For WC1 $(13 \lesssim G \lesssim 16)$ and WC2 $(G \gtrsim 16)$, the image is marginalised in the across-scan direction, retaining a one-dimensional array, of different lengths in WC1 and WC2, from which only the along-scan coordinate can be derived.

In the cyclic data-processing scheme adopted for Gaia (see Sect. 7.2 of Gaia Collaboration 2016), the astrometric global iterative solution (AGIS) is closely connected to a preceding step known as the intermediate data update (IDU). The IDU provides image centroids to AGIS, which are one- or two-dimensional depending on the WC; in turn, AGIS furnishes the IDU with the improved astrometry needed to calibrate the line- and pointspread functions used by the IDU for centroiding. AGIS and IDU are thus inseparable parts of a larger astrometric task, and the calibrations discussed below should be understood as including the relevant parts of the IDU calibrations, also done per WC.

The use of different calibration models for the three window classes effectively means that there are three separate instruments to calibrate, one for each $\mathrm{WC}^{7}$. Because the WC essentially depends on the magnitude of the source, most sources are always observed in the same WC. Disregarding, for the moment, the sources that are observed in more than one $\mathrm{WC}$, we have three disjoint subsets of sources, each subset being used to calibrate one WC. If the calibrations are allowed to be arbitrary functions of time, each subset could have its own reference frame, with an arbitrary offset in orientation and spin, without causing any inconsistency in the observations. All that is needed is that the time-dependent calibration of each WC at any time exactly matches the positional offsets of the sources in the corresponding subset, caused by the rotation of its frame. Although there is no particular reason why the calibration should vary in exactly this way, it is inevitable that unrelated variations caused by model imperfections contain some such component.

In reality, there is a good deal of overlap between the window classes, such that many sources around $G \simeq 13$ are sometimes observed in $\mathrm{WC} 0$, and other times in $\mathrm{WC1}$, and similarly around $G \simeq 16$ for WC1 and 2 . This should in principle suffice to guarantee a consistent reference frame across all magnitudes because it is not possible to obtain consistent solutions for the positions and proper motions of the multi-WC sources unless these parameters are expressed in the same reference frame for all the observations. Because there is no evidence of a discontinuity in spin at $G \simeq 16$ (Fig. 4 in Lindegren et al. 2018), the overlap indeed seems to have provided a good connection between the reference frames of WC1 and 2. As shown in the same figure, however, it did not work for the WC0/1 transition, or perhaps a more gradual change was created from $G \simeq 13$ to $\simeq 11$ (Sect. 3.4).

Of relevance here is that the modelling of observations in $\mathrm{WCO}$ is, for a number of reasons, much more difficult than in $\mathrm{WC} 1$ and 2. The two-dimensional images in $\mathrm{WCO}$ require the point-spread function to be modelled in both the along- and across-scan directions, in contrast to the simpler line-spread functions used in WC1 and 2. The across-scan profile has additional dependences on the precession-induced drift rate, resulting in a more complex calibration. Furthermore, to increase the dynamic range of Gaia's CCDs, stars brighter than $G \simeq 12$ normally obtain reduced integration time through the activation of TDI blocking gates (Crowley et al. 2016), and the gates also require separate calibrations. In spite of the gates, a substantial fraction of the $\mathrm{WC} 0$ observations are affected by pixel saturation, which causes additional issues. In Gaia DR2 the calibration of WC0 was in fact far from satisfactory, as is readily seen in a plot of the rms post-fit residual versus magnitude (Fig. 9 in Lindegren et al. 2018). This prevented a consistent astrometric modelling of the multi-WC sources across the WC0/1 boundary, which may have been the direct cause of the observed rotation.

To avoid a similar problem in future Gaia data releases, it is necessary to improve the modelling of WC0 observations. This is already part of ongoing activities towards the next releases. It will also help to maximise the number of multiWC sources in the primary astrometric solution. Additionally, the calibration models for the different window classes may be constrained not to contain any time-dependent components representing a rotation difference between the corresponding reference frames. Although these steps should ensure a consistent reference frame for all window classes, it remains important that the consistency can be confirmed, for example by means of VLBI observations.

\footnotetext{
7 In view of the extreme accuracy goals of Gaia, this is an inevitable consequence of using different observation modes. The use of TDI blocking gates, explained further below, means that there are in fact even more than three separate instruments to consider.
} 